\title{
UNIFORM CONVEXITY AND VARIATIONAL CONVERGENCE
}

\author{
V. V. ZHIKOV AND S. E. PASTUKHOVA
}

Dedicated to the Centennial Anniversary of B. M. Levitan

\begin{abstract}
Let $\Omega$ be a domain in $\mathbb{R}^{d}$. We establish the uniform convexity of the $\Gamma$-limit of a sequence of Carathéodory integrands $f(x, \xi): \Omega \times \mathbb{R}^{d} \rightarrow \mathbb{R}$ subjected to a two-sided power-law estimate of coercivity and growth with respect to $\xi$ with exponents $\alpha$ and $\beta, 1<\alpha \leq \beta<\infty$, and having a common modulus of convexity with respect to $\xi$. In particular, the $\Gamma$-limit of a sequence of power-law integrands of the form $|\xi|^{p(x)}$, where the variable exponent $p: \Omega \rightarrow[\alpha, \beta]$ is a measurable function, is uniformly convex.

We prove that one can assign a uniformly convex Orlicz space to the $\Gamma$-limit of a sequence of power-law integrands. A natural $\Gamma$-closed extension of the class of power-law integrands is found.

Applications to the homogenization theory for functionals of the calculus of variations and for monotone operators are given.
\end{abstract}

\section{INTRODUCTION}

1.1. The notion of uniformly convex Banach space was introduced by Clarkson in his famous paper [1. Recall that a norm $\|\cdot\|$ and the Banach space $V$ equipped with this norm are said to be uniformly convex if for each $\varepsilon \in(0,2)$ there exists a $\delta=\delta(\varepsilon)$ such that

$$
\|\xi-\eta\| \leq \varepsilon \quad \text { or } \quad\left\|\frac{\xi+\eta}{2}\right\| \leq 1-\delta
$$

for all $\xi, \eta \in V$ with $\|\xi\| \leq 1$ and $\|\eta\| \leq 1$. In this case, one also says that the unit ball is uniformly convex.

It is well known that the Euclidean norm

$$
|\xi|=\left(\xi_{1}^{2}+\cdots+\xi_{d}^{2}\right)^{1 / 2}
$$

on $\mathbb{R}^{d}$ is uniformly convex; this follows from the parallelogram identity

$$
|\xi-\eta|^{2}+|\xi+\eta|^{2}=2\left(|\xi|^{2}+|\eta|^{2}\right),
$$

which shows that one can set $\delta(\varepsilon)=1-\left(1-\varepsilon^{2}\right)^{1 / 2}$. (This has a simple geometric meaning for the unit sphere in $\mathbb{R}^{d}$ : the midpoints of chords of length $\geq \varepsilon \in(0,2)$ are at a distance of at least $\delta(\varepsilon)$ from the sphere.) The parallelogram identity also holds for the norm on a Hilbert space. As a consequence, every Hilbert space is uniformly convex.

The uniform convexity of other norms of the form

$$
\|\xi\|_{p}=\left(\left|\xi_{1}\right|^{p}+\cdots+\left|\xi_{d}\right|^{p}\right)^{1 / p}
$$

2010 Mathematics Subject Classification. Primary 35J20; Secondary 35J60, 46B10, 46B20, 49J45, $49 \mathrm{~J} 50$.

Key words and phrases. Uniform convexity, $\Gamma$-convergence, Orlicz spaces, power-law integrand, nonstandard coercivity, and growth conditions.

Supported by RFBR grant no. 14-01-00192a, grant no. NSh-3685.2014.1 of the President of the Russian Federation, and Russian Scientific Foundation grant no. 14-11-00398. 
on $\mathbb{R}^{d}$, where $1<p<2$ or $p>2$, as well as the norms on the spaces $l_{p}$ and $L^{p}(\Omega), p>1$, was proved in [1. The proof is based on the Clarkson inequalities

$$
\begin{array}{ll}
\|\xi-\eta\|^{p}+\|\xi+\eta\|^{p} \leq 2^{p-1}\left(\|\xi\|^{p}+\|\eta\|^{p}\right), & p \geq 2, \\
\|\xi-\eta\|^{q}+\|\xi+\eta\|^{q} \leq 2\left(\|\xi\|^{p}+\|\eta\|^{p}\right)^{q-1}, & q=\frac{p}{p-1}, \quad 1<p \leq 2,
\end{array}
$$

for these norms.

The norms $\|\xi\|_{1}=\left|\xi_{1}\right|+\cdots+\left|\xi_{d}\right|$ and $\|\xi\|_{\infty}=\max \left\{\left|\xi_{1}\right|, \ldots,\left|\xi_{d}\right|\right\}$ on $\mathbb{R}^{d}, d \geq 2$, are not uniformly convex. Neither are the Lebesgue spaces $L^{1}(\Omega)$ and $L^{\infty}(\Omega)$ as well as their discrete counterparts, the spaces $l_{1}$ and $l_{\infty}$.

A uniformly convex Banach space possesses a number of interesting, useful properties, of which we only note reflexivity (the Milman-Pettis theorem), the Banach-Saks property, and the uniform smoothness of the dual space; e.g., see [2, 3, 4, 5].

1.2. It is quite natural to consider not only uniformly convex sets but also uniformly convex functions. Recall that a function $f$ is said to be convex if

$$
f(\lambda \xi+(1-\lambda) \eta) \leq \lambda f(\xi)+(1-\lambda) f(\eta) \quad \forall \xi, \eta, \quad 0 \leq \lambda \leq 1,
$$

and strictly convex if the inequality in (1.1) is strict whenever $0<\lambda<1$ and $\xi \neq \eta$. As to uniform convexity, the following definition is classical.

Definition 1.1. A function $f$ defined on a Banach space $V$ is said to be uniformly convex if, for each $\varepsilon \in(0,1)$, there exists a $\delta=\delta(\varepsilon)>0$ such that

$$
\|\xi-\eta\| \leq \varepsilon \max \{\|\xi\|,\|\eta\|\} \quad \text { or } \quad 2 f\left(\frac{\xi+\eta}{2}\right) \leq(1-\delta)(f(\xi)+f(\eta))
$$

for any $\xi, \eta \in V$.

Clearly, a uniformly convex function is strictly convex. For $f(\xi)=\|\xi\|$, we obtain an equivalent definition of uniformly convex norm.

Uniformly convex functions have the property, important in applications, of strong convergence of a minimizing sequence.

Proposition 1.2. Let $f: V \rightarrow(-\infty,+\infty]$ be a uniformly convex lower semibounded function such that $\lim _{\|\xi\| \rightarrow \infty} f(\xi)=+\infty$. Let $A=\inf _{\xi \in V} f(\xi)$, and let $\xi_{n}$ be a minimizing sequence; i.e., $\lim _{n \rightarrow \infty} f\left(\xi_{n}\right)=A$. Then $\xi_{n}$ is a Cauchy sequence.

Proof. It follows from the properties of $f$ that the sequence $\xi_{n}$ is bounded (i.e., $\|\xi\| \leq M$ ) and that for each $\varepsilon \in(0,1)$ there exists a $\delta=\delta(\varepsilon)>0$ such that

$$
\left\|\xi_{n}-\xi_{n+m}\right\| \leq \varepsilon M \quad \text { or } \quad 2 f\left(\frac{\xi_{n}+\xi_{n+m}}{2}\right) \leq(1-\delta)\left(f\left(\xi_{n}\right)+f\left(\xi_{n+m}\right)\right)
$$

for any positive integers, $n$ and $m$. Since $\xi_{n}$ is a minimizing sequence, we see that $f\left(\xi_{n}\right)<A /(1-\delta)$ for sufficiently large $n, n \geq N(\varepsilon)$. Then the second inequality in (1.3) cannot hold for these $n$, because

$$
f\left(\frac{\xi_{n}+\xi_{n+m}}{2}\right) \geq A
$$

by the definition of $A$, while, on the other hand,

$$
(1-\delta)\left(f\left(\xi_{n}\right)+f\left(\xi_{n+m}\right)\right)<\frac{(1-\delta) 2 A}{1-\delta}=2 A .
$$

Thus, the first inequality in (1.3) holds for $n \geq N(\varepsilon)$, which means that $\xi_{n}$ is a Cauchy sequence. 
The definition of uniform convexity given above uses the norm. One can introduce uniform convexity for functions in a somewhat different way.

Definition 1.3. We say that a function $f: V \rightarrow(-\infty,+\infty]$ defined on a linear space $V$ is uniformly convex if for each $\varepsilon \in(0,1)$ there exists a $\delta=\delta(\varepsilon)>0$ such that

$$
f\left(\frac{\xi-\eta}{2}\right) \leq \varepsilon \frac{1}{2}(f(\xi)+f(\eta)) \quad \text { or } \quad f\left(\frac{\xi+\eta}{2}\right) \leq(1-\delta) \frac{1}{2}(f(\xi)+f(\eta))
$$

for any $\xi, \eta \in V$. The function $\delta(\varepsilon)$ is called the modulus of convexity.

With this approach, a uniformly convex function does not even have to be convex. In what follows, we touch upon the comparison of various types of convexity. As to uniform convexity, we usually stick to Definition 1.3, often without mentioning this explicitly.

1.3. We are interested in problems related to the uniform convexity of the vector OrliczLebesgue space (see Section 8 ). This is the space of measurable vector functions $v(x)$ such that the power $|v(x)|^{p(x)}$ with a given variable exponent $p(x)$ is integrable. The Orlicz-Lebesgue space is a natural generalization of the classical Lebesgue spaces. Being equipped with the Luxembourg norm, it becomes a uniformly convex Banach space. The Luxembourg norm is essentially the Minkowski functional of a convex set defined via an integral functional. This motivates our interest in the uniform convexity of integral functionals of the form

$$
\int_{\Omega} f(x, v(x)) d x, \quad \text { where } \Omega \text { is a domain in } \mathbb{R}^{d},
$$

and in the uniform convexity of the integrands $f(x, \xi), \xi \in \mathbb{R}^{d}$ (see Section 5).

The uniform convexity of functionals is of interest in that this property, as well as the usual convexity, is preserved under $\Gamma$-convergence. The definition of $\Gamma$-convergence and a discussion of its properties will be given in Sections 3 and 4

$\Gamma$-convergence is often referred to as variational convergence. The point is that this is a type of convergence $F_{\varepsilon} \rightarrow F$ of functionals which, under certain growth conditions on the functionals, implies the convergence of minima and minimizers in the corresponding variational problems

$$
\min _{v \in V} F_{\varepsilon}(v), \quad \text { where } V \text { is the space of admissible functions. }
$$

Similar passages to the limit in variational problems arise in various fields of nonlinear analysis, e.g., in homogenization theory and optimal control theory. The limit problem for the family (1.6) is

$$
\min _{v \in V} F(v), \quad F=\Gamma-\lim F_{\varepsilon} .
$$

It is important to find out what properties of $F_{\varepsilon}$ are inherited by the $\Gamma$-limit functional $F$. Uniform convexity is one of such properties (see Section 51).

Power-law functionals and their variational closure are of special interest owing to the search for a natural $\Gamma$-closed extension of this class (see Section 8).

\section{UNIFORM CONVEXITY OF FUNCTIONS ON $\mathbb{R}^{d}$}

We start from the power function $f(\xi)=|\xi|^{\alpha}, \xi \in \mathbb{R}^{d}$.

Proposition 2.1. The power function

$$
f(\xi)=|\xi|^{\alpha}, \quad \alpha>1,
$$

is uniformly convex. 
Proof. $1^{\circ}$. The power function $\varphi(t)=t^{\alpha}, t \in[0, \infty), \alpha>1$, on the real line is strictly convex (because $\varphi^{\prime \prime}(t)>0$ on $(0, \infty)$ ). This readily implies that the function $|\xi|^{\alpha}$ on $\mathbb{R}^{d}$ is strictly convex. (See $3^{\circ}$ below for details.)

$2^{\circ}$. Let us verify that the function $|\xi|^{\alpha}$ is uniformly convex in the sense of Definition 1.3 . Indeed, assume the contrary. Then for some $\varepsilon_{0} \in(0,1)$ there exist sequences of vectors $\xi^{n}$ and $\eta^{n}$ such that

$$
\left|\frac{\xi^{n}-\eta^{n}}{2}\right|^{\alpha} \geq \varepsilon_{0} \frac{1}{2}\left(\left|\xi^{n}\right|^{\alpha}+\left|\eta^{n}\right|^{\alpha}\right), \quad\left|\frac{\xi^{n}+\eta^{n}}{2}\right|^{\alpha} \geq\left(1-\delta_{n}\right) \frac{1}{2}\left(\left|\xi^{n}\right|^{\alpha}+\left|\eta^{n}\right|^{\alpha}\right),
$$

where $0<\delta_{n} \rightarrow 0$. Using homogeneity, one can assume that

$$
1=\left|\xi^{n}\right| \geq\left|\eta^{n}\right| \text {. }
$$

Then the sequences $\xi^{n}$ and $\eta^{n}$ are bounded and, hence, contain convergent subsequences; let $\bar{\xi}$ and $\bar{\eta}$ be their limit points. It follows from (2.2) and (2.3) that

$$
\left|\frac{\bar{\xi}-\bar{\eta}}{2}\right|^{\alpha} \geq \varepsilon_{0} \frac{1}{2}\left(1+|\bar{\eta}|^{\alpha}\right), \quad\left|\frac{\bar{\xi}+\bar{\eta}}{2}\right|^{\alpha} \geq \frac{1}{2}\left(|\bar{\xi}|^{\alpha}+|\bar{\eta}|^{\alpha}\right) .
$$

Relation $(2.4)_{2}$, together with the strict convexity of $|\xi|^{\alpha}$, implies that $\bar{\xi}=\bar{\eta}$, which is inconsistent with (2.4) $)_{1}$. This contradiction shows that the function $|\xi|^{\alpha}$ is uniformly convex.

$3^{\circ}$. It remains to verify the strict convexity of the function $|\xi|^{\alpha}, \alpha>1$, on $\mathbb{R}^{d}$. We need to prove the strict convexity inequality

$$
|\lambda \xi+(1-\lambda) \eta|^{\alpha}<\lambda|\xi|^{\alpha}+(1-\lambda)|\eta|^{\alpha} \quad \text { for all } \xi \neq \eta \text { and } 0<\lambda<1 .
$$

A homogeneity argument shows that it suffices to consider the case in which one of the vectors is of unit length. Let $\xi=e_{1}=(1,0, \ldots, 0)$ and $\eta=\left(\eta_{1}, \eta^{\prime}\right), \eta^{\prime} \in \mathbb{R}^{d-1}$, where $|\eta|^{2}=\left|\eta_{1}\right|^{2}+\left|\eta^{\prime}\right|^{2}$. If the vectors $\xi$ and $\eta$ are collinear (i.e., $\eta=s e_{1}, s \neq 1$ ), then inequality (2.5) follows from the corresponding strict convexity inequality for the real-valued function $t^{\alpha}$. If the vectors are not collinear, then $\eta^{\prime} \neq 0,\left|\eta_{1}\right|<|\eta|$, and, as a result,

$$
\begin{aligned}
\left|\lambda e_{1}+(1-\lambda) \eta\right|^{2} & =\left|\lambda+(1-\lambda) \eta_{1}\right|^{2}+(1-\lambda)^{2}\left|\eta^{\prime}\right|^{2} \\
& =\lambda^{2}+(1-\lambda)^{2}\left|\eta_{1}\right|^{2}+2 \lambda(1-\lambda) \eta_{1}+(1-\lambda)^{2}\left|\eta^{\prime}\right|^{2} \\
& =\lambda^{2}+(1-\lambda)^{2}|\eta|^{2}+2 \lambda(1-\lambda) \eta_{1} \\
& <\lambda^{2}+(1-\lambda)^{2}|\eta|^{2}+2 \lambda(1-\lambda)|\eta| \\
& =(\lambda+(1-\lambda)|\eta|)^{2} .
\end{aligned}
$$

Then

$$
\left|\lambda e_{1}+(1-\lambda) \eta\right|^{\alpha} \stackrel{\text { (2.6) }}{<}(\lambda+(1-\lambda)|\eta|)^{\alpha} \leq \lambda+(1-\lambda)|\eta|^{\alpha},
$$

where the last inequality holds by virtue of the usual convexity of the numerical function $t^{\alpha}$, as desired.

When proving Proposition 2.1, we have actually established (see $2^{\circ}$ ) the following assertion.

Proposition 2.2. Let $f: \mathbb{R}^{d} \rightarrow \mathbb{R}$ be a strictly convex function such that $f$ is positively homogeneous in $\xi$ (i.e., there exists an $s>0$ such that $f(k \xi)=k^{s} f(\xi)$ for all $k>0$ and $\xi \in \mathbb{R}^{d}$ ) and $f(\xi)=0$ only for $\xi=0$. Then $f(\xi)$ is uniformly convex in the sense of Definition 1.3 .

Let us give one more assertion like a test for uniform convexity. 
Proposition 2.3. Let $f: \mathbb{R}^{d} \rightarrow \mathbb{R}$ be a uniformly convex function, and let $\Phi: \mathbb{R} \rightarrow \mathbb{R}$ be a convex monotone increasing function with $\Phi(0)=0$. Then $\Phi(f(\xi))$ is uniformly convex with the same modulus of convexity as $f$.

Proof. We need to prove that the condition

$$
\Phi\left(f\left(\frac{\xi-\eta}{2}\right)\right)>\varepsilon \frac{\Phi(f(\xi))+\Phi(f(\eta))}{2}, \quad \varepsilon \in(0,1),
$$

implies the inequality

$$
\Phi\left(f\left(\frac{\xi+\eta}{2}\right)\right) \leq(1-\delta) \frac{\Phi(f(\xi))+\Phi(f(\eta))}{2}
$$

with the same $\delta$ as in (1.2). By the properties of $\Phi(t)$, condition (2.7) is possible only if

$$
f\left(\frac{\xi-\eta}{2}\right)>\varepsilon \frac{1}{2}(f(\xi)+f(\eta)) .
$$

Otherwise,

$$
\begin{aligned}
f\left(\frac{\xi-\eta}{2}\right) & \leq \varepsilon \frac{1}{2}(f(\xi)+f(\eta)) \\
& \Longrightarrow \Phi\left(f\left(\frac{\xi-\eta}{2}\right)\right) \leq \varepsilon \Phi\left(\frac{f(\xi)+f(\eta)}{2}\right) \leq \varepsilon \frac{\Phi(f(\xi))+\Phi(f(\eta))}{2}
\end{aligned}
$$

which contradicts (2.7). In the chain of inequalities $(2.10) 2$, we have used the monotonicity and convexity of $\Phi(t)$ (the latter implies that $\Phi(\varepsilon t) \leq \varepsilon \Phi(t)$, because $\Phi(0)=0$ ) at the first step and again the convexity of $\Phi(t)$ at the second step.

However, inequality (1.2) 2 holds under condition (2.9), and the same argument as in (2.10) leads to the desired inequality (2.8). The proof of the proposition is complete.

As a corollary, the following assertion is true.

Proposition 2.4. The power function $|\xi|^{p}$ with exponent $p \geq \alpha>1$ is uniformly convex with the same modulus of convexity $\delta=\delta(\varepsilon)$ as the power $|\xi|^{\alpha}$.

Indeed, it suffices to note that $|\xi|^{p}=\Phi\left(|\xi|^{\alpha}\right)$, where $\Phi(t)=t^{q}, q=p / \alpha \geq 1$, and apply Proposition 2.3 .

Remark. The uniform convexity of the function $|\xi|^{\alpha}$ for $\alpha \geq 2$ readily follows from the inequality

$$
|\xi+\eta|^{\alpha}+|\xi-\eta|^{\alpha} \leq 2^{\alpha-1}\left(|\xi|^{\alpha}+|\eta|^{\alpha}\right), \quad \xi, \eta \in \mathbb{R}^{d}, \quad \alpha \geq 2
$$

(which becomes the parallelogram identity for $\alpha=2$ ). In the case of $1<\alpha<2$, we do not have an equally simple inequality that would imply the uniform convexity of the function (2.1). That is why we have given a unified proof of the uniform convexity of the power function for all $\alpha>1$.

Let us give some comparison of various types of convexity.

Proposition 2.5. (i) Let $f: \mathbb{R}^{d} \rightarrow \mathbb{R}$ be a uniformly convex function in the sense of Definition 1.3 such that $f(\xi)=0$ only for $\xi=0$. Then $f$ is strictly convex.

(ii) Let $f(\xi)=\Phi(|\xi|)$, where $\Phi: \mathbb{R}_{+} \rightarrow \mathbb{R}$ is a convex monotone increasing function with $\Phi(0)=0$. Then the uniform convexity of $f$ in the sense of Definition 1.1 implies the uniform convexity in the sense of Definition 1.3 . 
Proof. The first claim can readily be proved by contradiction, and we omit the argument.

Let us prove the second claim. If $|\xi-\eta| \leq \varepsilon \max \{|\xi|,|\eta|\}$, then

$$
\frac{|\xi-\eta|}{2} \leq \varepsilon \frac{|\xi|+|\eta|}{2}
$$

By the properties of $\Phi$, we have

$$
\Phi\left(\frac{|\xi-\eta|}{2}\right) \leq \Phi\left(\varepsilon \frac{|\xi|+|\eta|}{2}\right) \leq \varepsilon \Phi\left(\frac{|\xi|+|\eta|}{2}\right) \leq \varepsilon \frac{1}{2}(\Phi(|\xi|)+\Phi(|\eta|))
$$

for $\varepsilon \in(0,1)$; i.e.,

$$
f\left(\frac{\xi-\eta}{2}\right) \leq \varepsilon \frac{1}{2}(f(\xi)+f(\eta))
$$

and the first part of the alternative (1.4) holds for the function $f(\xi)=\Phi(|\xi|)$. The second part of the alternative (1.4) coincides with what one has in (1.2).

\section{3. $\Gamma$-CONVERGENCE OF FUnCTIONS ON A METRIC SPACE AND THE PRESERVATION OF UNIFORM CONVEXITY}

3.1. By $V$ we denote a separable metric space with metric $\rho$. We equip the set of functions $F: V \rightarrow[-\infty,+\infty]$ with a special type of convergence, which is referred to as $\Gamma$-convergence, or variational convergence.

Definition 3.1. A function $\chi$ is a $\Gamma$-limit of a sequence of functions $F_{n}$ on $V$ if:

1. The lower semicontinuity condition

$$
\liminf _{n \rightarrow \infty} F_{n}\left(u_{n}\right) \geq \chi(u) \quad \text { whenever } u_{n} \rightarrow u
$$

is satisfied.

2. For each $u \in V$, there exists a sequence $u_{n} \rightarrow u$, which is called a $\Gamma$-realizing sequence, such that

$$
\lim _{n \rightarrow \infty} F_{n}\left(u_{n}\right)=\chi(u) .
$$

Selection principle. Every sequence of functions of $V$ contains a $\Gamma$-convergent subsequence.

A fairly comprehensive exposition of the theory of $\Gamma$-convergence can be found in 8 .

If the metric space $V$ has a linear structure consistent with the metric $\rho$, then one can consider functions with various convexity properties.

By $S_{\delta(\varepsilon)}$ we denote the class of uniformly convex functions on $V$ for which (1.4) holds with $\delta=\delta(\varepsilon)$.

Theorem 3.2. The class $S_{\delta(\varepsilon)}$ is closed with respect to $\Gamma$-convergence.

Proof. Let $F_{n} \stackrel{\Gamma}{\longrightarrow} \chi$, where the functions $F_{n}$ are uniformly convex and satisfy condition (1.4) with one and the same $\delta=\delta(\varepsilon)$. Let us prove that the same condition holds for the limit functional $\chi$. Fix arbitrary elements $u$ and $v$ and take $\Gamma$-realizing sequences

$$
\begin{aligned}
u_{n} & \rightarrow u, \quad v_{n} \rightarrow v \quad \text { in } V, \\
\lim _{n \rightarrow \infty} F_{n}\left(u_{n}\right) & =\chi(u), \quad \lim _{n \rightarrow \infty} F_{n}\left(v_{n}\right)=\chi(v) .
\end{aligned}
$$

Since

we have

$$
\frac{u_{n} \pm v_{n}}{2} \rightarrow \frac{u \pm v}{2} \quad \text { in } V
$$

$$
\liminf _{n \rightarrow \infty} F_{n}\left(\frac{u_{n} \pm v_{n}}{2}\right) \geq \chi\left(\frac{u \pm v}{2}\right)
$$


by property 1 (see Definition 3.1) of $\Gamma$-convergence of the functions $F_{n}$. Next, it follows from the uniform convexity of the functions $F_{n}$ that

$$
\begin{aligned}
& F_{n}\left(\frac{u_{n}-v_{n}}{2}\right) \leq \varepsilon \frac{1}{2}\left(F_{n}\left(u_{n}\right)+F_{n}\left(v_{n}\right)\right) \quad \text { or } \\
& F_{n}\left(\frac{u_{n}+v_{n}}{2}\right) \leq(1-\delta) \frac{1}{2}\left(F_{n}\left(u_{n}\right)+F_{n}\left(v_{n}\right)\right) .
\end{aligned}
$$

To verify the uniform convexity of the limit function $\chi$, we check that the condition

$$
\chi\left(\frac{u-v}{2}\right)-\varepsilon \frac{1}{2}(\chi(u)+\chi(v))=\tau>0
$$

implies the inequality

$$
\chi\left(\frac{u+v}{2}\right) \leq(1-\delta) \frac{1}{2}(\chi(u)+\chi(v)) .
$$

We use the limit relations (3.2) and (3.3) and write out the chain of inequalities (3.7)

$F_{n}\left(\frac{u_{n}-v_{n}}{2}\right) \stackrel{\text { (3.3) }}{\geq} \chi\left(\frac{u-v}{2}\right)-\frac{\tau}{3} \stackrel{\text { (3.5) }}{>} \varepsilon \frac{1}{2}(\chi(u)+\chi(v))+\frac{\tau}{3} \stackrel{\text { (3.2) }}{\geq} \varepsilon \frac{1}{2}\left(F_{n}\left(u_{n}\right)+F_{n}\left(v_{n}\right)\right)$ for sufficiently large $n, n>N(\tau)$. As a result,

$$
F_{n}\left(\frac{u_{n}-v_{n}}{2}\right)>\varepsilon \frac{1}{2}\left(F_{n}\left(u_{n}\right)+F_{n}\left(v_{n}\right)\right), \quad n>N(\tau),
$$

and we conclude by (3.4) that

$$
F_{n}\left(\frac{u_{n}+v_{n}}{2}\right) \leq(1-\delta) \frac{1}{2}\left(F_{n}\left(u_{n}\right)+F_{n}\left(v_{n}\right)\right), \quad n>N(\tau) .
$$

From this, by passing to the limit with regard for (3.2) and (3.3), we obtain the desired inequality (3.6).

Thus, we have proved the following alternative: for each $\varepsilon \in(0,1)$ and the corresponding $\delta=\delta(\varepsilon)$, one has

$$
\begin{aligned}
& \chi\left(\frac{u-v}{2}\right) \leq \varepsilon \frac{1}{2}(\chi(u)+\chi(v)) \quad \text { or } \\
& \chi\left(\frac{u+v}{2}\right) \leq(1-\delta) \frac{1}{2}(\chi(u)+\chi(v))
\end{aligned}
$$

for any $u, v \in V$, as desired.

3.2. Let us take the Sobolev space $W^{1, \alpha}(\Omega), \alpha>1$, for $V$, where $\Omega$ is a bounded domain with Lipschitz boundary in $\mathbb{R}^{d}$. Consider the class of functionals on $V$ satisfying the estimate

$$
F(u) \geq c_{1}\|\nabla u\|_{L^{\alpha}(\Omega)}^{\alpha}-c_{0}, \quad c_{1}>0, \quad c_{0} \geq 0 .
$$

For the preliminary definition of the $\Gamma$-limit, we equip $V=W^{1, \alpha}(\Omega)$ with the metric of the space $L^{1}=L^{1}(\Omega)$. It turns out that in this definition one can replace $L^{1}$-convergence by the weak convergence on $V$. As a result, we obtain the following definition.

Definition 3.3. A function $\chi$ is a $\Gamma$-limit of a sequence of functions $F_{n}$ on $V$ if:

1. The lower semicontinuity condition

$$
\liminf _{n \rightarrow \infty} F_{n}\left(u_{n}\right) \geq \chi(u) \quad \text { whenever } u_{n} \rightarrow u \text { in } V
$$

is satisfied. 
2. For each $u \in V$, there exists a sequence $u_{n} \in V$ such that

$$
u_{n} \rightarrow u \quad \text { in } V, \quad \lim _{n \rightarrow \infty} F_{n}\left(u_{n}\right)=\chi(u) .
$$

In the class (3.9), Definition 3.3 is clearly equivalent to the original definition of $\Gamma$ convergence with the $L^{1}$-metric. For example, let us show that property (3.1) of lower semicontinuity with respect to $L^{1}$-convergent sequences follows from property (3.10). Indeed, take $u_{n} \stackrel{L^{1}}{\rightarrow} u$ and consider two possibilities. If $\lim \inf _{n \rightarrow \infty}\left\|\nabla u_{n}\right\|_{L^{\alpha}}=\infty$, then $\liminf _{n \rightarrow \infty} F_{n}\left(u_{n}\right)=\infty$ by virtue of the estimate (3.9), and property (3.1) necessarily holds. If, on the contrary, the family $\nabla u_{n}$ is bounded in $L^{\alpha}$, then we automatically have $u_{n} \rightarrow u$ in $V$, and it suffices to use (3.10). Here we have used the fact that if $u_{n}$ is bounded in $W^{1, \alpha}(\Omega)$ and $u_{n} \rightarrow u$ in $L^{1}(\Omega)$, then $u_{n} \rightarrow u$ in $W^{1, \alpha}(\Omega)$.

In what follows, we consider $\Gamma$-convergence in the sense of Definition 3.3 .

\section{4. $\Gamma$-CONVERGENCE OF INTEGRANDS AND INHERITANCE OF SOME PROPERTIES}

4.1. Let $\Omega \subset \mathbb{R}^{d}$ be a bounded Lipschitz domain. We introduce the class of Carathéodory integrands $f(x, \xi): \Omega \times \mathbb{R}^{d} \rightarrow \mathbb{R}$ convex in $\xi$ and which satisfy the estimate

$$
-c_{0}+c_{1}|\xi|^{\alpha} \leq f(x, \xi) \leq c_{2}|\xi|^{\beta}+c_{0}, \quad c_{1}, c_{2}>0, \quad c_{0} \geq 0, \quad 1<\alpha \leq \beta<\infty .
$$

The corresponding integral functionals

$$
F(u)=\int_{\Omega} f(x, \nabla u) d x
$$

are considered on the Sobolev space $W^{1, \alpha}(\Omega)$, where they can take the value $+\infty$ if $\alpha<\beta$. One has the embedding

$$
W^{1, \beta}(\Omega) \subset \operatorname{dom} F=\left\{u \in W^{1, \alpha}(\Omega): F(u)<\infty\right\} .
$$

Obviously, $F$ is a convex functional weakly lower semicontinuous on $W^{1, \alpha}(\Omega)$. Moreover, $F$ is continuous on $W^{1, \beta}(\Omega)$ by virtue of the upper bound in (4.1).

We can define a relaxation functional (or relaxation) $\bar{F}$ by setting

$$
\bar{F}(u)=\inf _{u_{n} \in W^{1, \beta}(\Omega)} \lim _{u_{n} \rightarrow u} F\left(u_{n}\right), \quad u \in W^{1, \alpha}(\Omega) .
$$

Clearly, $F \leq \bar{F}$, and the inequality $F \neq \bar{F}$, closely related to what is known as Lavrent'ev's effect for variational problems, is possible.

For integrands of the class (4.1), we introduce $\Gamma$-convergence of two types.

Definition 4.1. An integrand $f$ is a $\Gamma_{1}$-limit of $f_{n}, f=\Gamma_{1}$-lim $f_{n}$, if

1. The lower semicontinuity condition

$$
\liminf _{n \rightarrow \infty} \int_{\Omega} f_{n}\left(x, \nabla u_{n}\right) d x \geq \int_{\Omega} f(x, \nabla u) d x \quad \text { whenever } u_{n} \rightarrow u \text { in } W^{1, \alpha}(\Omega)
$$

is satisfied.

2. For each $u \in W^{1, \beta}(\Omega)$, there exists a sequence $u_{n} \rightarrow u$ in $W^{1, \alpha}(\Omega)$ (referred to as a $\Gamma_{1}$-realizing sequence) such that

$$
\lim _{n \rightarrow \infty} \int_{\Omega} f_{n}\left(x, \nabla u_{n}\right) d x=\int_{\Omega} f(x, \nabla u) d x .
$$

The definition of $\Gamma_{2}$-convergence is obtained from that of $\Gamma_{1}$-convergence if one requires in both conditions that the approximating sequence $u_{n}$ belongs to the space $W^{1, \beta}(\Omega)$. This requirement proves to be rather important. The papers 9 and 7 give examples of sequences $f_{n}$ for which

$$
\Gamma_{1}-\lim f_{n} \neq \Gamma_{2}-\lim f_{n}
$$


The class (4.1) contains the power-law integrand

$$
f(x, \xi)=|\xi|^{p(x)}, \quad 1<\alpha \leq p(x) \leq \beta<\infty,
$$

where the variable exponent $p: \mathbb{R}^{d} \rightarrow \mathbb{R}$ is a measurable function. We often use this integrand in what follows.

4.2. We will use the theory of $\Gamma$-convergence of integrands given in [7] and [6]. The following assertion was proved in [6].

Compactness principle. For every sequence $f_{n}$ of integrands in the class (4.1), there exists a subsequence $f_{n^{\prime}}$ and an integrand $f$ in the class (4.1) such that $f_{n^{\prime}} \stackrel{\Gamma_{1}}{\longrightarrow} f$ in an arbitrary Lipschitz subdomain of $\Omega$.

A similar assertion holds for $\Gamma_{2}$-convergence.

We adopt the following convention: when speaking of $\Gamma_{1^{-}}$or $\Gamma_{2}$-limits in the domain $\Omega$, we always mean that the corresponding $\Gamma$-convergence of the integrands holds in an arbitrary Lipschitz subdomain of $\Omega$. With this approach, both $\Gamma$-limits are uniquely determined, as shown in [6]. It is this property that permits one to speak of the $\Gamma$ convergence of integrands rather than only of the $\Gamma$-convergence of integral functionals.

In what follows, we omit the subscript indicating the type of $\Gamma$-convergence whenever the assertion involved holds for both types of convergence.

The following principle is a useful tool for studying the $\Gamma$-convergence of integrands.

Locality principle. Let $f_{n}(x, \xi)$ be integrands of the class (4.1), let $f_{n} \stackrel{\Gamma}{\longrightarrow} f$, and let $u_{n} \rightarrow u$ be a $\Gamma$-realizing sequence. Then

$$
\lim _{n \rightarrow \infty} \int_{\Omega^{\prime}} f_{n}\left(x, \nabla u_{n}\right) d x=\int_{\Omega^{\prime}} f(x, \nabla u) d x,
$$

where $\Omega^{\prime}$ is an arbitrary Lipschitz subdomain of $\Omega$.

One can assign $\Gamma$-convergence in the sense of Definition 3.3 to a sequence of integral functionals of the form (4.2). There arise questions about the nature of the limit functional $\chi$ and its integral representation. Note that, in contrast to Definition 3.3. Definition 4.1 only uses integral functionals. Moreover, the existence of a $\Gamma$-realizing sequence is only required in Definition 4.1 for functions in the space $W^{1, \beta}(\Omega)$ (rather than $W^{1, \alpha}(\Omega)$, as in Definition 3.3. . Thus, there is no complete correspondence between the $\Gamma_{1}$-convergence of integrands of the class (4.1) and the $\Gamma$-convergence of the corresponding integral functionals. In the general case, if $F$ is an integral functional with integrand $f=\Gamma_{1}-\lim _{n \rightarrow \infty} f_{n}$, then

$$
F \leq \chi \leq \bar{F} \quad \text { on } W^{1, \alpha}(\Omega), \quad \chi=F \quad \text { on } W^{1, \beta}(\Omega),
$$

where $\bar{F}$ is the relaxation (see (4.3) ). We see that the abstract limit $\chi$ has an integral representation on $W^{1, \beta}(\Omega)$. We obtain a complete integral representation, namely, the relation $\chi=F$ on $W^{1, \alpha}(\Omega)$, provided that $F=\bar{F}$, which means that Lavrent'ev's effect is lacking. This happens, for example, for standard integrands (where $\alpha=\beta$ in (4.1)).

Instead of $\chi=\Gamma-\lim _{n \rightarrow \infty} F_{n}$, one can consider the limit in the sense of Definition 3.3 . namely, the $\Gamma$-limit $\tilde{\chi}=\Gamma-\lim _{n \rightarrow \infty} \bar{F}_{n}$ of relaxation functionals. Then the integral functional $F$ with integrand $f=\Gamma_{2}-\lim _{n \rightarrow \infty} f_{n}$ is related to $\tilde{\chi}$ by formulas like (4.8).

4.3. For $\Gamma$-convergence, the limit integrand inherits some properties, for example, convexity with respect to $\xi$, the two-sided power-law estimate (4.1), evenness with respect to $\xi(f(\cdot,-\xi)=f(\cdot, \xi))$, and the $\Delta_{2}$-condition

$$
\exists K>0: f(\cdot, \pm 2 \xi) \leq K f(\cdot, \xi) \quad \forall \xi .
$$

(An example of proof of these facts can be found in [10].) 
On the other hand, $\Gamma$-convergence preserves neither the power-law character of the integrands nor their isotropy. (An integrand $f(\cdot, \xi)$ is said to be isotropic if $f(\cdot, \xi)=f(\cdot, \eta)$ whenever $|\xi|=|\eta|$.) Counterexamples can be constructed with the help of homogenization theory (see [11, Chapter I]). It is well known that the homogenized integrand assigned in homogenization theory to a sequence of oscillating integrands is simultaneously the limit integrand from the viewpoint of $\Gamma$-convergence.

Example 1. Consider the homogenization problem for the sequence of integrands

$$
f(y, \xi)=\frac{|\xi|^{p(x / \varepsilon)}}{p(x / \varepsilon)},
$$

where $f(y, \xi)$ is a function periodic in $y$ with periodicity cell $\square=[0,1)^{d}$. It is well known that in the one-dimensional case $(d=1)$ the homogenized integrand has the representation

$$
\left(f^{\text {hom }}\right)^{*}(\xi)=\int_{0}^{1} \frac{|\xi|^{p^{\prime}(y)}}{p^{\prime}(y)} d y,
$$

where $g^{*}(\xi)=\sup _{\eta}\{\xi \cdot \eta-g(\eta)\}$ is the Young-Fenchel conjugate function. Take a piecewise constant exponent $p(y)$ taking two values $p_{1}$ and $p_{2}$. Then the integrand $\left(f^{\text {hom }}\right)^{*}(\xi)$ is a sum of power-law integrands with distinct exponents $p_{1}^{\prime}$ and $p_{2}^{\prime}$ Hölder conjugate to the original exponents $p_{1}$ and $p_{2}$, respectively. Hence, we see that the homogenized integrand $f^{\text {hom }}(\xi)$ itself is not power-law.

Example 2. Isotropy is not preserved under $\Gamma$-limits, as shown by examples of homogenization for the case of a quadratic integrand. Indeed, for $d=2$ consider the integrands

$$
f_{\varepsilon}(x, \xi)=a\left(\frac{x_{1}}{\varepsilon}\right)|\xi|^{2},
$$

where $a\left(y_{1}\right)>0$ is a continuous 1-periodic function with average $\langle a\rangle$ over the period. Homogenization theory says that [11, p. 12 of the Russian edition]

$$
\Gamma-\lim f_{\varepsilon}=\left\langle a^{-1}\right\rangle^{-1} \xi_{1}^{2}+\langle a\rangle \xi_{2}^{2}
$$

whence we see that the limit integrand is not isotropic.

Strict convexity is not preserved under $\Gamma$-convergence in the general case. However, it is preserved in the case of power-law integrands of the form (4.6) [12]. A power-law integrand is also uniformly convex. One can ask whether this important property is inherited under $\Gamma$-convergence. Later on in this paper (see Section 5), we show that the answer is "yes."

\section{UNIFORM CONVEXITY OF INTEGRANDS AND INTEGRAL FUNCTIONALS}

5.1. We are interested in integrands of the class (4.1) uniformly convex in $\xi$ and in the preservation of this property under $\Gamma$-convergence.

Definition 5.1. We say that an integrand $f$ of the class (4.1) belongs to the set $S_{\delta(\varepsilon)}=$ $S_{\alpha, \beta, \delta(\varepsilon)}$ if $f(\cdot,-\xi)=f(\cdot, \xi)$ and the alternative (1.4) holds with one and the same $\delta=\delta(\varepsilon)>0$ for any $\xi, \eta \in \mathbb{R}^{d}$ and for almost all $x \in \Omega$.

Lemma 5.2. If $f \in S_{\delta(\varepsilon)}$, then $F(u)$ is a uniformly convex functional with modulus of convexity

$$
\delta_{0}(\varepsilon)=\frac{\varepsilon}{2} \delta\left(\frac{\varepsilon}{2}\right) .
$$


Proof. Fix an $\varepsilon \in(0,1)$ and arbitrary functions $u, v \in \operatorname{dom} F$. Since the functional is convex and even, it follows that

$$
\frac{u+v}{2}, \frac{u-v}{2} \in \operatorname{dom} F
$$

Let us verify that the condition

$$
F\left(\frac{u-v}{2}\right)>\varepsilon \frac{1}{2}(F(u)+F(v))
$$

implies the inequality

$$
F\left(\frac{u+v}{2}\right) \leq(1-\delta) \frac{1}{2}(F(u)+F(v))
$$

with the above-mentioned $\delta_{0}=\delta_{0}(\varepsilon)>0$.

We introduce the set

$$
E=\left\{x \in \Omega: f\left(x, \frac{\nabla u-\nabla v}{2}\right)>\frac{\varepsilon}{2} \frac{f(x, \nabla u)+f(x, \nabla v)}{2}\right\} .
$$

Then

$$
f\left(x, \frac{\nabla u-\nabla v}{2}\right) \leq \frac{\varepsilon}{2} \cdot \frac{f(x, \nabla u)+f(x, \nabla v)}{2} \quad \text { on } E^{\prime}=\Omega \backslash E
$$

and

$$
F\left(\frac{u-v}{2}, E^{\prime}\right) \leq \frac{\varepsilon}{2} \cdot \frac{1}{2}\left(F\left(u, E^{\prime}\right)+F\left(v, E^{\prime}\right)\right) \leq \frac{\varepsilon}{2} \cdot \frac{1}{2}(F(u)+F(v)),
$$

whence it follows that

$$
F\left(\frac{u-v}{2}, E\right)=F\left(\frac{u-v}{2}\right)-F\left(\frac{u-v}{2}, E^{\prime}\right) \stackrel{5.1}{\geq} \frac{\varepsilon}{2} \cdot \frac{1}{2}(F(u)+F(v)) .
$$

Here and in the following, we write

$$
F(u, E)=\int_{E} f(x, \nabla u) d x .
$$

On the other hand, it follows from the choice of $E$ and the condition $f \in S_{\delta(\varepsilon)}$ that

$$
f\left(x, \frac{\nabla u+\nabla v}{2}\right) \leq\left(1-\delta_{2}\right) \cdot \frac{f(x, \nabla u)+f(x, \nabla v)}{2} \quad \text { on } E, \quad \delta_{2}=\delta\left(\frac{\varepsilon}{2}\right) ;
$$

consequently,

$$
F\left(\frac{u+v}{2}, E\right) \leq\left(1-\delta_{2}\right) \frac{1}{2}(F(u, E)+F(v, E)) .
$$

We write out the chain of inequalities

$$
\begin{aligned}
\frac{F(u)+F(v)}{2}- & F\left(\frac{u+v}{2}\right) \geq \frac{F(u, E)+F(v, E)}{2}-F\left(\frac{u+v}{2}, E\right) \\
& \stackrel{(5.7)}{\geq} \delta_{2} \frac{F(u, E)+F(v, E)}{2} \geq \delta_{2} F\left(\frac{u-v}{2}, E\right) \stackrel{(5.4)}{\geq} \frac{\varepsilon}{2} \delta_{2} \frac{F(u)+F(v)}{2},
\end{aligned}
$$

where convexity has been used at the first and third steps. (Namely,

$$
\begin{gathered}
\frac{1}{2}\left(F\left(u, E^{\prime}\right)+F\left(v, E^{\prime}\right)\right)-F\left(\frac{u+v}{2}, E^{\prime}\right) \geq 0, \\
\frac{1}{2}(F(u, E)+F(v, E)) \geq F\left(\frac{u-v}{2}, E\right),
\end{gathered}
$$


because $F(v)=F(-v)$ ). This chain implies the desired inequality (5.2) with

$$
\delta_{0}(\varepsilon)=\frac{\varepsilon}{2} \delta_{2}=\frac{\varepsilon}{2} \delta\left(\frac{\varepsilon}{2}\right)
$$

(see $\delta_{2}$ in $(5.6)$ ). The proof of the lemma is complete.

Remark 1. A local version of Lemma 5.2 holds as well. Namely, if $f \in S_{\delta(\varepsilon)}$, then for each $\varepsilon \in(0,1)$ there exists a $\delta_{0}(\varepsilon)>0$ (the same as before) such that

$$
\begin{aligned}
& F\left(\frac{u-v}{2}, E\right) \leq \varepsilon \frac{1}{2}(F(u, E)+F(v, E)) \text { or } \\
& F\left(\frac{u+v}{2}, E\right) \leq\left(1-\delta_{0}\right) \frac{1}{2}(F(u, E)+F(v, E))
\end{aligned}
$$

for any $u, v \in \operatorname{dom} F$, where $E$ is an arbitrary measurable set in $\Omega$.

Remark 2. The monograph 13] studied the relationship between the uniform convexity of the so-called generalized $N$-functions and of the corresponding functionals (see [13, Lemma 2.4.11]). However, the uniform convexity of $N$-functions is understood in [13] in a slightly different way than in the present paper. Furthermore, $N$-functions are defined on the real line and are suitable for scalar problems. The passage from vector functions to functionals is not completely obvious. Hence we are forced to prove Lemma 5.2, at the same time, much of the argument in 13 , has been used above.

5.2. Let us state the main result of this section.

Theorem 5.3. A $\Gamma$-limit of integrands of the class $S_{\delta(\varepsilon)}$ is a uniformly convex integrand with modulus of convexity

$$
\delta_{0}(\varepsilon)=\frac{\varepsilon}{2} \delta\left(\frac{\varepsilon}{2}\right) .
$$

Proof. Let $f=\Gamma-\lim _{n \rightarrow \infty} f_{n}$ and $f_{n} \in S_{\delta(\varepsilon)}$. Let us verify that $f \in S_{\delta_{0}(\varepsilon)}$ with $\delta_{0}(\varepsilon)$ indicated in the theorem.

By Lemma 5.2, the integral functionals $F_{n}$ corresponding to $f_{n}$ are uniformly convex with modulus of convexity $\delta_{0}(\varepsilon)$. Theorem 3.2 ensures the same property of the $\Gamma$-limit functional $\chi$. The latter coincides on $W^{1, \beta}(\Omega)$ with the integral functional $F$ corresponding to the $\Gamma$-limit integrand $f$ (see $\left.(4.8)_{2}\right)$. Thus, we have proved that for each $\varepsilon \in(0,1)$ and for the corresponding $\delta_{0}(\varepsilon)$ one has

$$
\begin{aligned}
& F\left(\frac{u-v}{2}\right) \leq \varepsilon \frac{1}{2}(F(u)+F(v)) \quad \text { or } \\
& F\left(\frac{u+v}{2}\right) \leq\left(1-\delta_{0}\right) \frac{1}{2}(F(u)+F(v))
\end{aligned}
$$

for any $u, v \in W^{1, \beta}(\Omega)$. Relations similar to (5.8) hold for an arbitrary Lipschitz subdomain $\Omega^{\prime} \subset \Omega$ by the locality principle (see Section 4) and Remark 1 above. Hence one can replace the functional $F(u)=F(u, \Omega)$ in (5.8) by $F\left(u, \Omega^{\prime}\right)$ (see notation (5.5)). In particular, if

$$
\begin{gathered}
\Omega^{\prime}=B_{r}\left(x_{0}\right) \quad \text { is a ball of radius } r>0 \text { centered at } x_{0}, \\
u, v \quad \text { are affine functions, } \quad \nabla u=\xi, \quad \nabla v=\eta,
\end{gathered}
$$

where $\xi, \eta \in \mathbb{R}^{d}$ are arbitrary, then either

$$
f_{B} f\left(x, \frac{\xi-\eta}{2}\right) d x \leq \varepsilon \frac{1}{2} f_{B}(f(x, \xi)+f(x, \eta)) d x
$$


or

$$
f_{B} f\left(x, \frac{\xi+\eta}{2}\right) d x \leq\left(1-\delta_{0}\right) \frac{1}{2} f_{B}(f(x, \xi)+f(x, \eta)) d x,
$$

where $B=B_{r}\left(x_{0}\right)$ and $f_{B} \cdot d x=|B|^{-1} \int_{B} \cdot d x$ is the average over the ball.

Assuming that $x_{0}$ is a Lebesgue point of the functions

$$
f(\cdot, \xi), \quad f(\cdot, \eta), \quad f\left(\cdot, \frac{\xi+\eta}{2}\right), \quad f\left(\cdot, \frac{\xi-\eta}{2}\right)
$$

(see Remark 3 below), from (5.9) and (5.10) we derive one of the following two pointwise relations for the integrand $f$ as the ball $B=B_{r}\left(x_{0}\right)$ shrinks to $x_{0}$ :

$$
\begin{aligned}
& f\left(x_{0}, \frac{\xi-\eta}{2}\right) \leq \varepsilon \frac{1}{2}\left(f\left(x_{0}, \xi\right)+f\left(x_{0}, \eta\right)\right) \quad \text { or } \\
& f\left(x_{0}, \frac{\xi+\eta}{2}\right) \leq\left(1-\delta_{0}\right) \frac{1}{2}\left(f\left(x_{0}, \xi\right)+f\left(x_{0}, \eta\right)\right),
\end{aligned}
$$

as desired.

More precisely, the passage to the limit in the alternative (5.9) $-(5.10)$ is based on the following argument. Assume that the opposite of inequality (5.11) 1 holds; i.e.,

$$
f\left(x_{0}, \frac{\xi-\eta}{2}\right)-\varepsilon \frac{1}{2}\left(f\left(x_{0}, \xi\right)+f\left(x_{0}, \eta\right)\right)=\tau>0 .
$$

Let us show that this implies inequality $(5.11)_{2}$ by virtue of (5.9) $-(5.10)$.

Indeed, by the property of Lebesgue points we have

$$
\begin{aligned}
f_{B} f\left(x, \frac{\xi-\eta}{2}\right) d x \geq & f\left(x_{0}, \frac{\xi-\eta}{2}\right)-\frac{\tau}{3} \\
& \stackrel{5.121)}{>} \varepsilon \frac{1}{2}\left(f\left(x_{0}, \xi\right)+f\left(x_{0}, \eta\right)\right)+\frac{\tau}{3} \geq \varepsilon \frac{1}{2} f_{B}(f(x, \xi)+f(x, \eta)) d x
\end{aligned}
$$

for the balls $B=B_{r}$ with sufficiently small $r, r<r_{0}(\tau)$. Thus, the inequality opposite to (5.9) holds on the balls $B=B_{r}, r<r_{0}(\tau)$. Consequently, it follows from the alternative (5.9) -(5.10) that inequality (5.10) holds on these balls, and we obtain the desired inequality (5.11) 2 in the limit as $r \rightarrow 0$ by the property of Lebesgue points. The proof of the theorem is complete.

Remark 3. Owing to convexity and the upper bound in (4.1), the following assertion holds for the integrands in question: there exists a set of full measure in $\Omega$ that consists of Lebesgue points of the function $f(\cdot, \xi)$ for every $\xi \in \mathbb{R}^{d}$.

\section{UNIFORM CONVEXITY OF CONJUGATE INTEGRANDS}

6.1. For a function $f: \mathbb{R}^{d} \rightarrow \mathbb{R}$, the conjugate function (the Young-Fenchel conjugate) is defined by

$$
f^{*}(\eta)=\sup _{\xi \in \mathbb{R}^{d}}[\eta \cdot \xi-f(\xi)] .
$$

It follows from the properties of conjugation (see [15, Section I.4] or [11, Chapter XII]) that the conjugate $f^{*}: \mathbb{R}^{d} \times \Omega \rightarrow \mathbb{R}$ of an integrand of the class (4.1) is a Carathéodory function convex in $\xi$ and satisfying the estimate

$$
-c_{0}+c_{1}^{*}|\xi|^{\beta^{\prime}} \leq f^{*}(x, \xi) \leq c_{2}^{*}|\xi|^{\alpha^{\prime}}+c_{0}, \quad c_{1}^{*}, c_{2}^{*}>0,
$$

where the prime stands for the Hölder conjugate exponents. 
One can readily verify that the conjugate of the power-law integrand

$$
f(x, \xi)=\frac{|\xi|^{p(x)}}{p(x)}, \quad p(x)>1,
$$

is again the power-law integrand

$$
f^{*}(x, \xi)=\frac{|\xi|^{p^{\prime}(x)}}{p^{\prime}(x)}, \quad p^{\prime}(x)=\frac{p(x)}{p(x)-1} .
$$

The convergence $f_{n} \stackrel{\Gamma}{\longrightarrow} f$ preserves some properties of conjugate integrands, e.g., uniform convexity. In what follows, we use the class $S_{\beta^{\prime}, \alpha^{\prime}, \delta(\varepsilon)}^{*}=S_{\delta(\varepsilon)}^{*}$ of conjugate integrands defined by analogy with the class $S_{\alpha, \beta, \delta(\varepsilon)}$ (see Definition [5.1).

Theorem 6.1. Let $f_{n}$ be integrands of the class (4.1), let $f_{n} \stackrel{\Gamma}{\longrightarrow} f$, and let $f_{n}^{*} \in$ $S_{\beta^{\prime}, \alpha^{\prime}, \delta(\varepsilon)}^{*}$. Then the conjugate $f^{*}$ of the limit integrand is uniformly convex, and moreover, $f^{*} \in S_{\beta^{\prime}, \alpha^{\prime}, \delta_{0}(\varepsilon)}$, where $\delta_{0}(\varepsilon)=\frac{\varepsilon}{2} \delta\left(\frac{\varepsilon}{2}\right)$.

The proof of Theorem 6.1 will be given in Section 7

6.2. Let us present an important application of Theorem 6.1 to the study of differentiability of $\Gamma$-limit integrands. It is based on the fact that differentiability and strict convexity for functions on $\mathbb{R}^{d}$ are dual to each other via Young-Fenchel conjugation; see Proposition 6.5 below.

Recall that if an integrand $f(x, \xi)$ is differentiable in $\xi$, then the corresponding integral functional $F(u)$ of the form (4.2) is Gâteaux differentiable, and one can write out the Euler equation for the minimizer in the variational problem. This is the differential equation

$$
\operatorname{div}_{x}\left(\left.\nabla_{\xi} f(x, \xi)\right|_{\xi=\nabla u}\right)=0,
$$

where $\nabla_{\xi} f(x, \xi)$ is the differential of the function $f(x, \xi)$ with respect to $\xi$. For the power-law integrand

we have

$$
f(x, \xi)=\frac{|\xi|^{p(x)}}{p(x)}
$$

$$
\nabla_{\xi} f(x, \xi)=|\xi|^{p(x)-2} \xi
$$

and the Euler equation contains the $p(x)$-Laplacian, which is a natural generalization of the ordinary Laplacian. In the general case where the integrand $f(x, \xi)$ is convex but not differentiable in $\xi$, the Euler equation is given in terms of the subdifferential implicitly, because the latter is not unique.

Now let $f(x, \xi)$ be the $\Gamma$-limit of a sequence of power-law integrands $f_{n}(x, \xi)$. Is $f(x, \xi)$ differentiable with respect to $\xi$, and can one write out the Euler equation with an explicit differential operator of the type of the $p(x)$-Laplacian, as in (6.3)? In what follows, we show that the answer is "yes."

Definition 6.2. The class $P(\alpha, \beta)$ is the $\Gamma$-closure of the set of power-law integrands $|\xi|^{p(x)}$ with measurable exponent $p: \mathbb{R}^{d} \rightarrow[\alpha, \beta], 1<\alpha \leq \beta<\infty$.

In fact, for $\alpha \neq \beta$, we obtain two distinct classes corresponding to $\Gamma_{1^{-}}$and $\Gamma_{2^{-}}$ convergence (see the definitions in Section 4), and one can consider either of them.

Theorem 6.3. The integrands $f(x, \xi)$ of the class $P(\alpha, \beta)$ and the conjugate integrands $f^{*}(x, \xi)$ are differentiable with respect to $\xi$.

This theorem can be derived from the following two assertions. 
Proposition 6.4. The integrands $f(x, \xi)$ of the class $P(\alpha, \beta)$ and the conjugate integrands $f^{*}(x, \xi)$ are uniformly convex in $\xi$ in the sense of Definition 1.3 .

Proposition 6.5. The following conditions are equivalent for a convex function $f: \mathbb{R}^{d} \rightarrow$ $\mathbb{R}:$ (i) $f$ is differentiable; (ii) $f^{*}$ is strictly convex.

The latter assertion is well known in convex analysis (e.g., see [14, Section 1.19]). Let as make some remarks concerning the proof of Theorem 6.3

First, by Proposition 2.4. power-law integrands of the form (4.6) satisfy condition (1.2) with one and the same function $\delta(\varepsilon)$ corresponding to the power $|\xi|^{\alpha}$, and hence Theorem 5.3 applies to this family of integrands.

Second, power-law integrands, as well as $\Gamma$-limits of power-law integrands, vanish only at $\xi=0$. (For the latter, this positivity property is studied in Section 8 see Proposition 8.2) As a consequence, uniform convexity in the class $P(\alpha, \beta)$ automatically implies strict convexity (see Proposition 2.5). The same is true for the integrands conjugate to integrands of the class $P(\alpha, \beta)$. Then, by Proposition 6.5, one can claim that integrands of the class $P(\alpha, \beta)$ are differentiable with respect to $\xi$.

\section{Procedure for finding the $\Gamma$-Limit integrand AND PRESERVATION OF UNIFORM CONVEXITY}

In this section, we prove Theorem 6.1. The proof of the similar Theorem 5.3 (where the inheritance of uniform convexity by the limit $f$ itself was considered) used the technique of $\Gamma$-realizing sequences. To reveal the properties of the conjugate integrand $f^{*}$, we employ a different method based on the procedure for finding the $\Gamma$-limit suggested in [6]. At all stages of this procedure, we verify that uniform convexity is preserved for the auxiliary objects in terms of which $f^{*}$ is defined.

7.1. Recall the procedure for finding the $\Gamma$-limit.

$1^{\circ}$. For an integrand $f(x, \xi)$ of the class (4.1), we define the following function, which is called the local characteristic in what follows:

$$
\varphi_{1}(\xi)=\varphi_{1}(B, \xi)=\min _{u \in W_{0}^{1, \alpha}(B)} f_{B} f(x, \xi+\nabla u) d x,
$$

where $f_{B} \cdot d x=\frac{1}{|B|} \int_{B} \cdot d x$ is the average over a cube $B \subset \Omega$. The subscript in $\varphi_{1}(\xi)$ indicates that the local characteristic handles $\Gamma_{1}$-convergence. To simplify the notation, we omit this subscript and write $\varphi(\xi)$ instead of $\varphi_{1}(\xi)$.

Obviously, the convex problem (7.1) has a solution by virtue of the left estimate in (4.1). By standard methods, using the minimizer in problem (7.1), we show that the local characteristic is convex and satisfies the estimate

$$
-c_{0}+c_{1}|\xi|^{\alpha} \leq \varphi(\xi) \leq c_{2}|\xi|^{\beta}+c_{0} .
$$

$2^{\circ}$. In what follows, we consider closed cubes in $\mathbb{R}^{d}$ with edges parallel to coordinate axes and with vertices at rational points. The symbol $\mathcal{B}$ stands for an arbitrary set that is the union of finitely many such cubes with pairwise disjoint interiors; i.e.,

$$
\mathcal{B}=\bigcup_{j} B_{j}, \quad \operatorname{int}\left(B_{j} \cap B_{i}\right)=\emptyset .
$$

Set $\lambda(\mathcal{B})=\max \left\{h_{j}\right\}$, where $h_{j}$ is the edge length of the cube $B_{j}$.

Definition 7.1. A decomposition of $\Omega$ into cubes is a family $\mathcal{B}_{r}$ of the form (7.3) such that (i) $\lambda\left(\mathcal{B}_{r}\right) \rightarrow 0$ as $r \rightarrow 0$; (ii) $\left|\Omega \backslash \mathcal{B}_{r}\right| \rightarrow 0$ as $r \rightarrow 0$. 
$3^{\circ}$. Recall some facts about convex functions.

If $A(u)$ is a convex function on a Banach space, then

$$
\left|A\left(u_{1}\right)-A\left(u_{2}\right)\right| \leq R^{-1} L\left\|u_{1}-u_{2}\right\|, \quad\left\|u_{1}\right\|,\left\|u_{2}\right\| \leq R, \quad L=\sup _{\|u\| \leq 2 R}|A(u)|
$$

i.e., a convex function bounded on a ball of radius $2 R$ is Lipschitz on the ball of radius $R$. This implies the following compactness properties:

(C1) The class of convex functions $\varphi(\xi)\left(\xi \in \mathbb{R}^{d}\right)$ satisfying the estimate (7.2) is compact in the sense of uniform convergence on every compact subset of $\mathbb{R}^{d}$.

(C2) Every sequence of integrands $f_{k}(x, \xi)$ of the class (4.1) contains a subsequence $f_{k^{\prime}}(x, \xi)$ weakly convergent in $L^{2}(\Omega)$ to $f(x, \xi)$ as $k^{\prime} \rightarrow \infty$ for each $\xi \in \mathbb{R}^{d}$.

$4^{\circ}$. Let us proceed to the construction of $\Gamma_{1}-\lim _{n \rightarrow \infty} f_{n}$ itself. We use formula (7.1) to define the local characteristics $\varphi_{n}(B, \xi)$ of the integrands $f_{n}$. By property $(\mathrm{C} 1)$, we can assume that there exists a limit

$$
\lim _{n \rightarrow \infty} \varphi_{n}(B, \xi)=\varphi(B, \xi)
$$

for every cube $B$ with rational vertices and every $\xi \in \mathbb{R}^{d}$.

Consider an arbitrary decomposition of $\mathcal{B}_{r}$ into cubes, and set

$$
\Phi_{r}(x, \xi)= \begin{cases}\varphi(B, \xi) & \text { if } x \in B \subset \mathcal{B}_{r}, \\ -c_{0}+c_{1}|\xi|^{\alpha} & \text { if } x \in \Omega \backslash \mathcal{B}_{r} .\end{cases}
$$

Obviously, the family $\Phi_{r}(x, \xi)$ belongs to the class (4.1), and it follows from property (C2) that there exists an integrand

$$
f(x, \xi)=\lim _{r \rightarrow 0} \Phi_{r}(x, \xi) \quad \text { for all } \xi \in \mathbb{R}^{d}
$$

defined in the sense of weak convergence in $L^{2}(\Omega)$.

As was shown in [6], the integrand $f(x, \xi)$ constructed according to the scheme (7.4)(17.6) is the $\Gamma_{1}$-limit of the sequence of integrands $f_{n}(x, \xi)$ and, as well as the family $f_{n}$ itself, belongs to the class (4.1). Moreover, the following assertion holds.

Proposition 7.2. The convergence (17.6) is strong in $L^{2}(\Omega)$ and can be viewed as pointwise convergence for almost all $x \in \Omega$.

$5^{\circ}$. To construct the $\Gamma_{2}$-limit integrand, we use the local characteristic $\varphi_{2}(\xi)=$ $\varphi_{2}(B, \xi)$, which is defined via the variational problem

$$
\varphi_{2}(\xi)=\inf _{u \in W_{0}^{1, \beta}(B)} f_{B} f(x, \xi+\nabla u) d x
$$

Since the exponents $\alpha$ and $\beta$ in the coercivity and growth condition (4.1) can be distinct, it follows that the local characteristics $\varphi_{1}$ and $\varphi_{2}$ do not coincide in general. One always has $\varphi_{1} \leq \varphi_{2}$, and the strict inequality is possible. This is a manifestation of the so-called Lavrent'ev effect: the minimization of one and the same functional over all admissible functions and over only smooth admissible functions gives distinct results.

Convexity and the two-sided estimate (7.1) can be verified for $\varphi_{2}$ in the same way as for $\varphi_{1}$ but with the use of $\delta$-minimizers. The infimum in problem (7.7) may not be attained, there may exist no minimizer, and one has to work with a minimizing sequence. After that, the procedure (7.4)-(7.6) is reproduced without modifications. 
7.2. Let us introduce the dual local characteristics $\psi(\xi)=\psi(B, \xi)$ of two types

$$
\psi_{1}(\xi)=\inf _{v \in V_{\mathrm{sol}}^{\beta^{\prime}}(B)} f_{B} g(x, \xi+v) d x, \quad \psi_{2}(\xi)=\inf _{v \in V_{\mathrm{sol}}^{\alpha^{\prime}}(B)} f_{B} g(x, \xi+v) d x,
$$

where $g(x, \xi)$ is the conjugate of the integrand $f(x, \xi)$ (see 6.10$), B \subset \Omega$ is a cube, and

$$
V_{\text {sol }}^{\gamma}(B)=\left\{v \in L^{\gamma}(B)^{d}: \operatorname{div} v=0, \int_{B} v d x=0\right\}, \quad 1<\gamma<\infty,
$$

is the space of solenoidal vectors in $L^{\gamma}(B)^{d}$ with zero average.

There exists a one-to-one correspondence between the characteristics $\varphi_{1}, \varphi_{2}$ (see (7.1) and (7.7) ) and $\psi_{1}, \psi_{2}$, which is established by the duality theorem in convex analysis.

Lemma 7.3. One has the relations

$$
\varphi_{1}=\psi_{2}^{*}, \quad \varphi_{2}=\psi_{1}^{*}, \quad \psi_{1}=\varphi_{2}^{*}, \quad \psi_{2}=\varphi_{1}^{*}
$$

The variational representation of the characteristics $\psi(\xi)$ has some corollaries: first, these are convexity and the two-sided estimate

$$
c_{1}^{*}|\xi|^{\beta^{\prime}}-c_{0} \leq \psi(\xi) \leq c_{2}^{*}|\xi|^{\alpha^{\prime}}+c_{0}
$$

of the type of (6.2); second, the local characteristic $\psi(\xi)$ inherits uniform convexity from $g$.

Lemma 7.4. If $g \in S_{\delta(\varepsilon)}^{*}$, then $\psi(\xi)$ is a uniformly convex function with modulus of convexity $\delta_{0}(\varepsilon)=\frac{\varepsilon}{2} \delta\left(\frac{\varepsilon}{2}\right)$.

Proof. Just as in Lemma 5.2, one can claim that the integral functional

$$
G(w)=G(w, B)=f_{B} g(x, w) d x, \quad w \in L^{\beta^{\prime}}(B)^{d},
$$

is uniformly convex. More precisely, for each $\varepsilon \in(0,1)$, one has the implication

$$
G\left(\frac{w_{1}-w_{2}}{2}\right)>\varepsilon \frac{1}{2}\left(G\left(w_{1}\right)+G\left(w_{2}\right)\right) \Longrightarrow G\left(\frac{w_{1}+w_{2}}{2}\right) \leq\left(1-\delta_{0}\right) \frac{1}{2}\left(G\left(w_{1}\right)+G\left(w_{2}\right)\right)
$$

with $\delta_{0}=\delta_{0}(\varepsilon)>0$ indicated in the lemma, where $w_{1}, w_{2} \in L^{\beta^{\prime}}(B)^{d}$ are arbitrary.

Now we need to show that for each $\varepsilon \in(0,1)$ and for $\delta_{0}=\delta_{0}(\varepsilon)$ one has the implication

$$
\psi\left(\frac{\xi_{1}-\xi_{2}}{2}\right)>\varepsilon \frac{1}{2}\left(\psi\left(\xi_{1}\right)+\psi\left(\xi_{2}\right)\right) \Longrightarrow \psi\left(\frac{\xi_{1}+\xi_{2}}{2}\right) \leq\left(1-\delta_{0}\right) \frac{1}{2}\left(\psi\left(\xi_{1}\right)+\psi\left(\xi_{2}\right)\right)
$$

for any $\xi_{1}, \xi_{2} \in \mathbb{R}^{d}$, which just means the uniform convexity of the function $\psi(\xi)$.

In what follows, to be definite, we set $\psi=\psi_{1}$. Then

$$
\begin{aligned}
\psi(\xi)=f_{B} g\left(x, \xi+v_{\xi}\right) d x & =f_{B} g\left(x, w_{\xi}\right) d x \\
\stackrel{(7.111)}{=} G\left(w_{\xi}\right) & \leq f_{B} g(x, \xi+v) d x \quad \forall v \in V_{\mathrm{sol}}^{\beta^{\prime}}(B),
\end{aligned}
$$

$v_{\xi}$ is a minimizer in problem (7.8) $1, \quad w_{\xi}=\xi+v_{\xi}$.

Let $\xi_{1}, \xi_{2}$ be such that inequality $(7.13)_{1}$ is satisfied, which, with regard for the representation (7.14), can be rewritten as

$$
G\left(w_{\frac{\xi_{1}-\xi_{2}}{2}}\right)>\varepsilon \frac{1}{2}\left(G\left(w_{\xi_{1}}\right)+G\left(w_{\xi_{2}}\right)\right) .
$$


On the other hand, by inequality (17.14) 4 we have

$$
G\left(w_{\frac{\xi_{1}-\xi_{2}}{2}}\right) \leq G\left(\frac{\xi_{1}-\xi_{2}}{2}+\frac{v_{\xi_{1}}-v_{\xi_{2}}}{2}\right)=G\left(\frac{w_{\xi_{1}}-w_{\xi_{2}}}{2}\right),
$$

where $w_{\xi_{1}}=\xi_{1}+v_{\xi_{1}}$ and $w_{\xi_{2}}=\xi_{2}+v_{\xi_{2}}$. Inequalities (7.15) and (7.16) imply the inequality

$$
G\left(\frac{w_{\xi_{1}}-w_{\xi_{2}}}{2}\right)>\varepsilon \frac{1}{2}\left(G\left(w_{\xi_{1}}\right)+G\left(w_{\xi_{2}}\right)\right),
$$

and we conclude by (7.12) that

$$
G\left(\frac{w_{\xi_{1}}+w_{\xi_{2}}}{2}\right) \leq \frac{1}{2}\left(1-\delta_{0}\right)\left(G\left(w_{\xi_{1}}\right)+G\left(w_{\xi_{2}}\right)\right) .
$$

On the other hand,

$$
G\left(\frac{w_{\xi_{1}}+w_{\xi_{2}}}{2}\right)=G\left(\frac{\xi_{1}+\xi_{2}}{2}+\frac{v_{\xi_{1}}+v_{\xi_{2}}}{2}\right) \stackrel{\frac{7.14}{\geq}}{\geq} G\left(\frac{\xi_{1}+\xi_{2}}{2}+v_{\frac{\xi_{1}+\xi_{2}}{2}}\right)=G\left(w_{\frac{\xi_{1}+\xi_{2}}{2}}\right) .
$$

We represent the right-hand sides of inequalities (7.17) and (7.18) via the local characteristic and obtain

$$
\psi\left(\frac{\xi_{1}+\xi_{2}}{2}\right) \leq\left(1-\delta_{0}\right) \frac{1}{2}\left(\psi\left(\xi_{1}\right)+\psi\left(\xi_{2}\right)\right) .
$$

Thus, we have obtained inequality $(\underline{7.13})_{2}$, as desired. The proof of the lemma is complete.

7.3. Proof of Theorem 6.1. Set $g_{n}=f_{n}^{*}$, where $f_{n} \stackrel{\Gamma}{\longrightarrow} f$. In Section [7.1, we have presented a procedure for finding the limit integrand $f=\Gamma-\lim _{n \rightarrow \infty} f_{n}$. Let us define a limit procedure related to $g_{n}$ which gives the conjugate integrand $f^{*}$ in the limit and extends the uniform convexity of the integrands $g_{n}$ to their limit. We will use the following assertion, whose proof can be found, say, in [12].

Lemma 7.5. Assume that

(i) $f_{n}(\xi)$ is convex with respect to $\xi \in \mathbb{R}^{d}$.

(ii) $-c_{0}+c_{1}|\xi|^{\alpha} \leq f_{n}(\xi) \leq c_{2}|\xi|^{\beta}+c_{0}, 1<\alpha \leq \beta, c_{0} \geq 0$, and $c_{1}, c_{2}>0$. If $f_{n}(\xi) \rightarrow f(\xi)$ for all $\xi$, then $f_{n}^{*}(\eta) \rightarrow f^{*}(\eta)$ for all $\eta$.

To the integrands $g_{n}$, we assign the local characteristics $\psi_{n}(\xi)=\psi_{n}(B, \xi)$. By Lemma 7.5. the pointwise convergence (17.4) implies the pointwise convergence $\psi_{n} \rightarrow$ $\psi=\varphi^{*}$.

For the decomposition $\mathcal{B}_{r}$ of $\Omega$ into cubes, we define

$$
\Psi_{r}(x, \xi)= \begin{cases}\psi(B, \xi) & \text { if } x \in B \subset \mathcal{B}_{r} \\ -c_{0}+c_{1}^{*}|\xi|^{\beta^{\prime}} & \text { if } x \in \Omega \backslash \mathcal{B}_{r}\end{cases}
$$

Let us prove the pointwise convergence

$$
\lim _{r \rightarrow 0} \Psi_{r}=f^{*} .
$$

Indeed, $\Psi_{r}=\left(\Phi_{r}\right)^{*}$ by construction (see (7.5) and (7.19) $)$. We again use Lemma 7.5 to derive the pointwise convergence of conjugate functions, i.e., relation (17.20), from the pointwise convergence $\Phi_{r}(x, \xi) \rightarrow f(x, \xi)$ (see Proposition 7.2) .

By Lemma 7.4, the functions $\psi_{n}$ are uniformly convex. The pointwise passage to the limit preserves this property first for $\psi$ (and hence for $\Psi_{r}(x, \cdot)$ ) and then also for $f^{*}$. This completes the proof of Theorem 6.1 


\section{ON ORLICZ SPACES}

8.1. Definitions and main properties. By $L^{p(\cdot)}(\Omega)$ we denote the class of measurable functions $v: \Omega \rightarrow \mathbb{R}^{d}$ such that

$$
A_{p}(v)=\int_{\Omega}|v(x)|^{p(x)} d x<\infty
$$

where the exponent $p(x)$ is the same as in (4.6). This class becomes a reflexive Banach space if we equip it with the norm

$$
\|v\|_{L^{p(\cdot)}(\Omega)}=\inf \left\{\lambda>0: A_{p}\left(\lambda^{-1} v\right) \leq 1\right\}
$$

referred to as the Luxembourg norm. Note that the infimum in (8.2) is attained if $A_{p}(v)>0$. Furthermore,

$$
\|v\|_{L^{p(\cdot)}(\Omega)}=\lambda>0 \Longleftrightarrow A_{p}\left(\lambda^{-1} v\right)=1
$$

This implies the unit ball property

$$
\|v\|_{L^{p(\cdot)}(\Omega)} \leq 1 \Leftrightarrow A_{p}(v) \leq 1
$$

Along with $L^{p(\cdot)}(\Omega)$, let us introduce the generalized Orlicz space $L^{f}(\Omega)$ as the class of measurable functions $v: \Omega \rightarrow \mathbb{R}^{d}$ such that

$$
A_{f}(v)=\int_{\Omega} f(x, v(x)) d x<\infty
$$

with the Luxembourg norm

$$
\|v\|_{L^{f}(\Omega)}=\inf \left\{\lambda>0: A_{f}\left(\lambda^{-1} v\right) \leq 1\right\} .
$$

The space $L^{f}(\Omega)$ is well defined provided that for the integrand $f(x, \xi)$ in (8.5) one takes functions satisfying the following conditions:

$(\mathcal{N}) f(x, \xi): \Omega \times \mathbb{R}^{d} \rightarrow \mathbb{R}_{+}$is a Carathéodory function.

It is convex and even in $\xi$.

It satisfies the $\Delta_{2}$-condition (4.9).

One has $f(\cdot, \xi)=0$ only for $\xi=0$.

The norm $\|\cdot\|_{L^{f}}$ retains many of the properties of the norm $\|\cdot\|_{L^{p(\cdot)}}$, in particular, the unit ball property. Clearly, the power-law integrand (4.6) belongs to the class $(\mathcal{N})$.

Theorem 8.1. Let an integrand $f$ of the class $(\mathcal{N})$ be uniformly convex in the sense of Definition 1.3. Then the Orlicz space $L^{f}(\Omega)$ is uniformly convex as well. In particular, the Lebesgue-Orlicz space $L^{p(\cdot)}(\Omega)$ is uniformly convex.

This theorem is deemed to be well known in some form. To make the exposition self-contained, we give some comments on the proof in Section 10 .

8.2. On the $\Gamma$-closure of some sets of integrands. Let us discuss some questions related to the $\Gamma$-closure on the set of integrands of the class $(\mathcal{N})$ satisfying the estimate (4.1). The set itself is not $\Gamma$-closed. The point is that the last property in the list $(\mathcal{N})$ is not controlled by anything under $\Gamma$-closure. Let us replace this property by a stronger one, which is already preserved in the $\Gamma$-limit. Consider the integrands satisfying the two-sided estimate

$$
m(\xi) \leq f(\cdot, \xi) \leq M(\xi) \quad \text { for all } \xi \in \mathbb{R}^{d},
$$

where the positive functions $M(\xi)$ and $m(\xi)$ are chosen as follows:

$$
M(\xi)=\max \left\{|\xi|^{\alpha},|\xi|^{\beta}\right\},
$$

$m(\xi)$ is the convex hull of the function $\min \left\{|\xi|^{\alpha},|\xi|^{\beta}\right\}$.

The estimate (8.7) holds, say, for the power-law integrand (4.6). 
Both functions in (8.8) are convex, and one can use the weak lower semicontinuity of convex functionals (see [7]). Then the preservation of the estimate (8.7) in the $\Gamma$-limit can be shown with the use of the technique of $\Gamma$-realizing sequences (see its application in Sections 3 and 5 . The same approach or the procedure for finding the $\Gamma$-limit integrand (see Section 7) can be used to verify the preservation of the other properties in the list $(\mathcal{N})$. Let us summarize our considerations.

Proposition 8.2. The set of integrands of the class $(\mathcal{N})$ satisfying the estimate (8.7) is closed under $\Gamma$-convergence.

8.3. On a $\Gamma$-closed extension of the class of power-law integrands. Consider the variational closure of the power-law integrands $f(x, \xi)=|\xi|^{p(x)}$ in more detail. Let us introduce the following real-valued functions of the argument $t \geq 0$ :

$$
\begin{aligned}
& \mu_{1}(t)=\min _{p \in[\alpha, \beta]} t^{p}, \quad \mu_{2}(t)=\max _{p \in[\alpha, \beta]} t^{p}, \\
& \mu_{1}(t)=\left\{\begin{array}{ll}
t^{\alpha} & \text { if } t \geq 1, \\
t^{\beta} & \text { if } 0 \leq t \leq 1,
\end{array} \quad \mu_{2}(t)= \begin{cases}t^{\beta} & \text { if } t \geq 1, \\
t^{\alpha} & \text { if } 0 \leq t \leq 1 .\end{cases} \right.
\end{aligned}
$$

Now for $\lambda>0$ we have

$$
\begin{aligned}
f(x, \xi) & =f\left(x, \lambda^{-1} \xi\right) \lambda^{p(x)}, \\
\mu_{1}(\lambda) f\left(x, \lambda^{-1} \xi\right) & \leq f(x, \xi) \leq \mu_{2}(\lambda) f\left(x, \lambda^{-1} \xi\right), \\
\mu_{1}(\lambda) A_{f}\left(\lambda^{-1} v\right) & \leq A_{f}(v) \leq \mu_{2}(\lambda) A_{f}\left(\lambda^{-1} v\right),
\end{aligned}
$$

whence for $\lambda=\|v\|_{f}$ we obtain

$$
\mu_{1}\left(\|v\|_{f}\right) \leq A_{f}(v) \leq \mu_{2}\left(\|v\|_{f}\right) .
$$

In the simplest case of $f(x, \xi)=|\xi|^{p}, p=$ const, relation (8.10) becomes

$$
\int_{\Omega}|v|^{p} d x=\|v\|_{L^{p}(\Omega)}^{p} .
$$

The inequalities stated above for a power-law integrand are preserved under $\Gamma$-closure, which can be proved by the method of $\Gamma$-realizing sequences.

Proposition 8.3. Let $f$ be the $\Gamma$-limit of a sequence of power-law integrands of the form (4.6). Then one has the estimate (8.9) and, as a consequence, the estimates (8.10) and (8.11).

Thus, the set of integrands satisfying the estimates (8.9) can be viewed as a natural $\Gamma$-closed extension of the class of power-law integrands. It is this estimate, rather than the more general estimate (8.7), that ensures relation (8.11), characteristic of power-law integrands, between the Luxembourg norm and the corresponding integral functional.

8.4. Sobolev-Orlicz spaces and $\boldsymbol{\Gamma}$-convergence of the norms. We introduce the Sobolev-Orlicz space

$$
W^{f}=\left\{u \in W^{1, \alpha}(\Omega), \nabla u \in L^{f}\right\}
$$

with the norm

$$
\|u\|_{W^{f}}=\|\nabla u\|_{L^{f}}+\|u\|_{L^{\alpha}}
$$

The norm functional $\|u\|_{W^{f}}$ can be assumed to be defined on the entire space $V=$ $W^{1, \alpha}(\Omega)$; to this end, we set it to be $+\infty$ on $W^{1, \alpha}(\Omega) \backslash W^{f}$. 
Theorem 8.4. Let $f_{n}$ and $f$ be integrands of the class $(\mathcal{N})$ satisfying the estimate (4.1), and let $F_{n} \stackrel{\Gamma}{\longrightarrow} F$. Then the corresponding Sobolev-Orlicz norms $\Gamma$-converge; i.e.,

$$
\|\cdot\|_{W^{f_{n}}} \stackrel{\Gamma}{\longrightarrow}\|\cdot\|_{W^{f}} .
$$

Proof. In what follows, we use the simplified notation $\|\cdot\|_{n}$ and $\|\cdot\|$ for the norms on $L^{f_{n}}$ and $L^{f}$, respectively, and denote the functionals (8.5) with integrands $f_{n}$ and $f$ by $A_{n}(\cdot)$ and $A(\cdot)$, respectively.

Let us verify the lower semicontinuity of the convergence (8.13). Let

$$
u_{n} \rightarrow u \text { in } V, \quad v_{n}=\nabla u_{n}, \quad v=\nabla u .
$$

In view of the structure of the norm (8.12), it suffices to establish the relation

$$
\liminf _{n \rightarrow \infty}\left\|v_{n}\right\|_{n} \geq\|v\|
$$

because $\left\|u_{n}\right\|_{L^{\alpha}} \rightarrow\|u\|_{L^{\alpha}}$ by the embedding theorem.

Without loss of generality, we assume that the sequence $\lambda_{n}=\left\|v_{n}\right\|_{n}$ has a finite limit, $\lambda_{n} \rightarrow \tilde{\lambda}$, so that

$$
\frac{u_{n}}{\lambda_{n}} \rightarrow \frac{u}{\widetilde{\lambda}} \text { in } V, \quad 1=\int_{\Omega} f_{n}\left(x, \frac{v_{n}}{\lambda_{n}}\right) d x .
$$

This, by virtue of condition 1 for the convergence $F_{n} \stackrel{\Gamma}{\longrightarrow} F$ and the unit ball property, implies that

$$
1 \geq \int_{\Omega} f\left(x, \frac{v}{\widetilde{\lambda}}\right) d x, \quad\|v\| \leq \widetilde{\lambda}
$$

which gives the desired inequality (8.14).

It is somewhat harder to verify property 2 in the definition of $\Gamma$-convergence of the norm functionals. For a given $u \in W^{f}$, we need to find

$$
\begin{aligned}
u_{n} \in W^{f_{n}}, & u_{n} \rightarrow u \quad \text { in } W^{1, \alpha}(\Omega), \\
\left\|v_{n}\right\|_{n} \rightarrow\|v\|, & v_{n}=\nabla u_{n}, \quad v=\nabla u,
\end{aligned}
$$

since $\left\|u_{n}\right\|_{L^{\alpha}} \rightarrow\|u\|_{L^{\alpha}}$ automatically by the embedding theorem. Without loss of generality, we assume that $\|v\|=1$ and hence $A(v)=1$. By virtue of the convergence $F_{n} \stackrel{\Gamma}{\longrightarrow} F$, there exists a sequence $v_{n}=\nabla u_{n}$ such that

$$
A_{n}\left(v_{n}\right)=F_{n}\left(u_{n}\right) \rightarrow F(u)=A(v)=1 .
$$

The desired norm convergence $\left\|v_{n}\right\|_{n} \rightarrow\|v\|$ follows from the lemma given below. This completes the proof of Theorem 8.4

Lemma 8.5. Let $f_{n}$ be integrands of the class $(\mathcal{N})$. If $A_{n}\left(v_{n}\right) \rightarrow 1$, then $\left\|v_{n}\right\|_{n} \rightarrow 1$.

Proof. Assume the contrary: There exists an $\varepsilon_{0}>0$ and a subsequence, which will be denoted again by $\{n\}$ in what follows, such that the norm $\lambda_{n}=\left\|v_{n}\right\|_{n}>0$ satisfies one of the following two consditions:

$$
\lambda_{n} \geq 1+\varepsilon_{0} \quad \text { or } \quad \lambda_{n} \leq 1-\varepsilon_{0} .
$$

Then it follows from the property of the Luxembourg norm and convexity that either

$$
1=A_{n}\left(\frac{v_{n}}{\lambda_{n}}\right) \leq \frac{1}{\lambda_{n}} A_{n}\left(v_{n}\right)<\frac{1}{1+\varepsilon_{0}} A_{n}\left(v_{n}\right)
$$

under condition $(8.15)_{1}$ or

$$
1=A_{n}\left(\frac{v_{n}}{\lambda_{n}}\right) \geq \frac{1}{\lambda_{n}} A_{n}\left(v_{n}\right)>\frac{1}{1-\varepsilon_{0}} A_{n}\left(v_{n}\right)
$$


under condition $(8.15)_{2}$. Since $A_{n}\left(v_{n}\right) \rightarrow 1$, we arrive at a contradiction in either case. The proof of the lemma is complete.

Remark. Theorem 8.4 assumes the $\Gamma$-convergence of the integral functionals $F_{n}$ to the integral functional $F$, which is not always the case given the $\Gamma$-convergence $f_{n} \stackrel{\Gamma}{\longrightarrow} f$ of the integrands. This problem about the integral representation of the limit functional $\Gamma$ - $\lim F_{n}=\chi$ via the limit integrand $\Gamma$ - $\lim f_{n}=f$, i.e., the problem as to whether $\chi=F$, was discussed in Section 4.2. The relation $F_{n} \stackrel{\Gamma}{\longrightarrow} F$ holds if the functional $F$ coincides with its relaxation $\bar{F}$, which is the case, say, for the standard integrands ( $\alpha=\beta$ in (4.1) $)$ or when averaging oscillating integrands (see Section 9.1).

\section{Supplement: HomogenizAtion EXAmples}

Here we present examples of limit objects, such as integrands, functionals, and Orlicz spaces, which inherit the uniform convexity of the original objects under homogenization.

9.1. Homogenization of functionals in calculus of variations. Let $f(y, \xi)$ be an integrand of the class (4.1) periodic in $y \in \mathbb{R}^{d}$, and let $\square=\left[-\frac{1}{2}, \frac{1}{2}\right)^{d}$ be the periodicity cell. Set $f_{\varepsilon}(x, \xi)=f\left(\frac{x}{\varepsilon}, \xi\right), \varepsilon>0$, and define the homogenized integrands

$$
\begin{aligned}
f_{1}^{\text {hom }}(\xi) & =\min _{u \in W_{\text {per }}^{1, \alpha}(\square)} \int_{\square} f(x, \xi+\nabla u) d x, \\
f_{2}^{\text {hom }}(\xi) & =\inf _{u \in W_{\text {per }}^{1, \beta}(\square)} \int_{\square} f(x, \xi+\nabla u) d x,
\end{aligned}
$$

where $W_{\text {per }}^{1, \gamma}(\square)$ is the Sobolev space of periodic functions. Then

$$
\Gamma_{i^{-}} \lim _{\varepsilon \rightarrow 0} f_{\varepsilon}=f_{i}^{\text {hom }}, \quad i=1,2,
$$

and obviously $f_{1}^{\text {hom }} \leq f_{2}^{\text {hom }}$. There is a known example (see 9]) of an integrand for which $f_{1}^{\text {hom }} \neq f_{2}^{\text {hom }}$. The integrand has the form $|\xi|^{p(y)}, y, \xi \in \mathbb{R}^{2}$, where $p(y)$ is periodic and is given by

$$
p(y)=\left\{\begin{array}{ll}
\alpha & \text { if } y_{1} y_{2}>0, \\
\beta & \text { if } y_{1} y_{2}<0,
\end{array} \quad \alpha<2<\beta,\right.
$$

on the periodicity cell $\square=\left[-\frac{1}{2}, \frac{1}{2}\right)^{2}$.

Since the homogenized integrands are the limit integrands in the sense of $\Gamma$-convergence (see (9.2)), it follows from Theorem 5.3 that each of them is uniformly convex provided that so is the original integrand $f(y, \xi)$ if, moreover, $f(y, \xi)$ is even in $\xi$. For example, one can take the power-law integrand $f(y, \xi)=|\xi|^{p(y)}$. In the last case, the integrands $f_{i}^{\text {hom }}(\xi)$ are differentiable as well by Theorem 6.3 .

Note yet another peculiarity of the homogenized integrands: they are independent of the space variable. Owing to this property, the homogenized integral functional coincides with the relaxation of itself [15, so that Theorem 8.4 applies. 17.

Functionals with oscillating integrands of the form $f\left(\frac{x}{\varepsilon}, u, \nabla u\right)$ were studied in $[16$,

9.2. Homogenization of monotone operators. Consider the homogenization of the Dirichlet problem

$$
\left\{\begin{array}{l}
\operatorname{div} A\left(\frac{x}{\varepsilon}, \nabla u_{\varepsilon}\right)=\operatorname{div} g \quad \text { in } \Omega, \\
\left.u_{\varepsilon}\right|_{\partial \Omega}=0, \quad g \in L^{\infty}(\Omega)^{d} .
\end{array}\right.
$$


Here $\Omega \subset \mathbb{R}^{d}$ is a bounded Lipschitz domain, and $A(y, \xi): \mathbb{R}^{d} \times \mathbb{R}^{d} \rightarrow \mathbb{R}^{d}$ is a Carathéodory vector function periodic in $y$ with periodicity cell $\square=\left[-\frac{1}{2}, \frac{1}{2}\right)^{d}$ such that

$$
\begin{aligned}
(A(y, \xi)-A(y, \eta)) \cdot(\xi-\eta) & >0 \quad \text { for almost all } y \in \square \text { and } \xi \neq \eta, \\
A(y, \xi) \cdot \xi & \geq c_{0}\left(|\xi|^{p(y)}-1\right), \\
|A(y, \xi)|^{p^{\prime}(y)} & \leq c_{1}\left(|\xi|^{p(y)}+1\right), \quad p^{\prime}(y)=\frac{p(y)}{p(y)-1},
\end{aligned}
$$

where the measurable exponent $p$ is periodic with the same periodicity cell $\square$ and

$$
1<\alpha \leq p(y) \leq \beta<\infty .
$$

We seek a solution of problem (9.4) in the Sobolev-Orlicz space corresponding to the exponent $p_{\varepsilon}(x)=p(x / \varepsilon)$. We define this space as

$W_{0}^{1, p_{\varepsilon}(\cdot)}(\Omega)=\left\{u \in W_{0}^{1,1}(\Omega), \int_{\Omega}|\nabla u|^{p_{\varepsilon}(x)} d x<\infty\right\}, \quad\|u\|_{W_{0}^{1, p_{\varepsilon}(\cdot)}(\Omega)}=\|\nabla u\|_{L^{p_{\varepsilon}(\cdot)}(\Omega)}$.

Definition 9.1. A function $u_{\varepsilon} \in W_{0}^{1, p_{\varepsilon}(\cdot)}$ is called a solution of problem (9.4) if the identity

$$
\int_{\Omega} A\left(\frac{x}{\varepsilon}, \nabla u_{\varepsilon}\right) \cdot \nabla \varphi d x=\int_{\Omega} g \cdot \nabla \varphi d x
$$

holds for an arbitrary test function $\varphi \in W_{0}^{1, p_{\varepsilon}(\cdot)}(\Omega)$.

Note the following important fact: smooth functions are not necessarily dense in Sobolev-Orlicz spaces, in contrast to the classical Sobolev spaces. For example, in Definition 9.1 it is insufficient to require that the integral identity (9.9) holds only for $\varphi \in C_{0}^{\infty}(\Omega)$. It is this situation that arises if one takes the exponent $p(y)$ defined in (9.3). In this connection, we define the space $H_{0}^{1, p_{\varepsilon}(\cdot)}(\Omega)$ as the closure of $C_{0}^{\infty}(\Omega)$ in $W_{0}^{1, p_{\varepsilon}(\cdot)}(\Omega)$.

Definition 9.2. A function $u_{\varepsilon} \in H_{0}^{1, p_{\varepsilon}(\cdot)}(\Omega)$ is called an $H$-solution if identity (9.9) holds for an arbitrary test function $\varphi \in H_{0}^{1, p_{\varepsilon}(\cdot)}(\Omega)$. (It suffices to require this only for $\varphi \in C_{0}^{\infty}(\Omega)$.)

A solution in the sense of Definition 9.1 will be called a $W$-solution.

The existence and uniqueness of a $W$ - or an $H$-solution of problem (9.4) under assumptions (9.5)-(9.8) can be proved by the method of monotone operators [18. In this case, the following uniform estimate holds for the solutions and fluxes:

$$
\int_{\Omega}\left|\nabla u_{\varepsilon}\right|^{p_{\varepsilon}(x)} d x \leq c, \quad \int_{\Omega}\left|A\left(\frac{x}{\varepsilon}, \nabla u_{\varepsilon}\right)\right|^{p_{\varepsilon}^{\prime}(x)} d x \leq c,
$$

where the constant only depends on the constants $c_{0}$ and $c_{1}$ in conditions (9.6) and (9.7) and on $\|g\|_{L^{\alpha^{\prime}}(\Omega)}$. One can assume that there is a weak convergence of the solutions and fluxes,

$$
u_{\varepsilon} \rightarrow u \quad \text { in } W_{0}^{1, \alpha}(\Omega), \quad A\left(\frac{x}{\varepsilon}, \nabla u_{\varepsilon}\right) \rightarrow z \quad \text { in } L^{\beta^{\prime}}(\Omega)^{d} .
$$

It readily follows from (9.9) that

$$
\int_{\Omega} z \cdot \nabla \varphi d x=\int_{\Omega} g \cdot \nabla \varphi d x
$$

for $\varphi \in C_{0}^{\infty}(\Omega)$. We need to find the relation between the limit elements $u$ and $z$. As is customary in homogenization theory [11, this relation should have the form

$$
z=a(\nabla u),
$$


where the symbol $a(\xi)$ is already independent from the space variable. Relations (9.12) and (9.13) specify the homogenized equation

$$
\operatorname{div}(a(\nabla u))=\operatorname{div} g .
$$

However, how should one understand this equation? Is it related to a well-posed problem? In what space is this problem defined? How does one "homogenize" as $\varepsilon \rightarrow 0$ the "oscillating" spaces $W_{0}^{1, p_{\varepsilon}(\cdot)}(\Omega)$ and $H_{0}^{1, p_{\varepsilon}(\cdot)}(\Omega)$ occurring in the statement of the boundary value problem (9.4)? In what follows, we answer these questions. (See [19] for details.)

The limit elements in (9.11) in general depend on the choice of the type of solution, so that we have two homogenized symbols $a_{1}(\xi)$ and $a_{2}(\xi)$. The homogenized symbols $a_{1}(\xi)$ and $a_{2}(\xi)$ are defined by

$$
a_{i}(\xi)=\int_{\square} A\left(y, \xi+\nabla N_{i}\right) d y, \quad i=1,2,
$$

where $N_{1}$ and $N_{2}$ are solutions of the following problems on the periodicity cell:

$$
\begin{array}{lll}
N_{1} \in W_{\text {per }}^{1, p(\cdot)}(\square), \quad & \int_{\square} A\left(y, \xi+\nabla N_{1}\right) \cdot \nabla \varphi d y=0 & \forall \varphi \in W_{\text {per }}^{1, p(\cdot)}(\square), \\
N_{2} \in H_{\text {per }}^{1, p(\cdot)}(\square), \quad & \int_{\square} A\left(y, \xi+\nabla N_{2}\right) \cdot \nabla \varphi d y=0 \quad \forall \varphi \in C_{\text {per }}^{\infty}(\square) .
\end{array}
$$

Here we use the Sobolev-Orlicz spaces of periodic functions,

$$
\begin{gathered}
W_{\text {per }}^{1, p(\cdot)}(\square)=\left\{u \in W_{\text {per }}^{1,1}(\square), \int_{\square} u d y=0, \int_{\square}|\nabla u|^{p} d y<\infty\right\}, \\
H_{\text {per }}^{1, p(\cdot)}(\square) \text { is the closure of } C_{\text {per }}^{\infty}(\square) \text { in } W_{\text {per }}^{1, p(\cdot)}(\square) .
\end{gathered}
$$

The norm on $W_{\text {per }}^{1, p(\cdot)}(\square)$ is given by $\|u\|_{W_{\text {per }}^{1, p(\cdot)}(\square)}=\|\nabla u\|_{L^{p(\cdot)}(\square)}$.

The homogenized symbols (9.14) inherit coercivity and boundedness (see (9.6) and (9.7) ), but this happens in a rather peculiar way, via the model homogenized integrands $f_{1}$ and $f_{2}$ determined from the variational problem

$$
\begin{aligned}
& f_{1}(\xi)=f_{1}^{\mathrm{hom}}(\xi)=\min _{u \in W_{\mathrm{per}}^{1, p(\cdot)}(\square)} \int_{\square} \frac{|\xi+\nabla u|^{p(y)}}{p(y)} d y, \\
& f_{2}(\xi)=f_{2}^{\mathrm{hom}}(\xi)=\min _{u \in H_{\mathrm{per}}^{1, p(\cdot)}(\square)} \int_{\square} \frac{|\xi+\nabla u|^{p(y)}}{p(y)} d y .
\end{aligned}
$$

The integrands $f_{1}$ and $f_{2}$ can substantially differ from power functions of the form $|\xi|^{\alpha}$, and hence the homogenized problem is solved in the generalized Sobolev-Orlicz spaces related to $f_{1}$ and $f_{2}$ rather than in the classical Sobolev spaces. Let us explain this last thing in more detail.

One can readily verify that $f_{1}$ and $f_{2}$ are functions of the class $(\mathcal{N})$ (see Section 8). Thus, we have the Orlicz space $L^{f}=L^{f}(\Omega)$ and the corresponding Sobolev-Orlicz spaces

$$
W_{0}^{f}=W_{0}^{f}(\Omega)=\left\{u \in W_{0}^{1,1}(\Omega), f(\nabla u) \in L^{1}(\Omega)\right\}, \quad\|u\|_{W_{0}^{f}}=\|\nabla u\|_{L^{f}},
$$

where one can take $f_{1}$ or $f_{2}$ for $f$.

Let us list the main properties of the vector function $a(\xi)$ (equal to $a_{1}$ or $a_{2}$ in (9.14)) in which the integrands in (9.15) are used. They are as follows:

(i) Continuity on $\mathbb{R}^{d}$.

(ii) Strict monotonicity, $\left(a\left(\xi_{1}\right)-a\left(\xi_{2}\right)\right) \cdot\left(\xi_{1}-\xi_{2}\right)>0$ for $\xi_{1} \neq \xi_{2}$.

(iii) Coercivity, $a(\xi) \cdot \xi \geq c_{0} f(\xi)-1, c_{0}>0$. 
(iv) "Boundedness from above," $f^{*}(a(\xi)) \leq c_{1} f(\xi)+1$, where $f^{*}(\eta)$ is the YoungFenchel conjugate function; see (6.1).

Using the estimates (9.10) and the weak lower semicontinuity of the convex functional with the power-law integrand $|\xi|^{p_{\varepsilon}(x)}[9$, we prove that the limit elements in (9.11) belong to the respective limit spaces, $u \in W_{0}^{f}$ and $z \in L^{f^{*}}$. These spaces possess the important uniform convexity property (see Section 8).

Now we are in a position to refine the statement of the homogenized problem.

Homogenized problem. Find a $u \in W_{0}^{1,1}(\Omega)$ such that

$$
\begin{gathered}
f(\nabla u) \in L^{1}(\Omega), \quad f^{*}\left(a(\nabla u) \in L^{1}(\Omega),\right. \\
\operatorname{div} a(\nabla u)=\operatorname{div} g \quad \text { (in the sense of distributions on } \Omega \text { ). }
\end{gathered}
$$

In fact, here we have two homogenized problems for the pairs $a_{1}, f_{1}$ and $a_{2}, f_{2}$.

Using the properties of the symbol $a(\xi)$, one can prove the existence and uniqueness of a solution of the homogenized problem for every $g \in L^{f^{*}}(\Omega)$ by the monotone operator method.

\section{Supplement: Proof of Theorem 8.1}

We use the following assertions, which refine the unit ball property in the Orlicz space $L^{f}(\Omega)$ :

(i) For each $\varepsilon \in(0,1)$ there exists a $\delta=\delta(\varepsilon)$ such that $A_{f}(v) \leq \delta \Rightarrow\|v\|_{L^{f}} \leq \varepsilon$.

(ii) For each $\varepsilon \in(0,1)$ there exists a $\delta=\delta(\varepsilon)>0$ such that $A_{f}(v) \leq 1-\varepsilon \Rightarrow\|v\|_{L^{f}} \leq$ $1-\delta$.

Their proof is given at the end of the section. If the integrand $f$ is power-law or belongs to the class $P(\alpha, \beta)$ (see Definition 6.2), then the estimate (8.11) is satisfied, which readily implies (i) and (ii).

Lemma 10.1. If the integrand $f$ is uniformly convex, then $A_{f}(\cdot)$ is a uniformly convex functional.

We omit the proof, which is similar to that of Lemma 5.2

To simplify the notation, we write $X=L^{f}(\Omega)$ and $\|\cdot\|=\|\cdot\|_{L^{f}}$. Fix an $\varepsilon \in(0,1)$ and arbitrary

$$
u, v \in X: \quad\|u\| \leq 1, \quad\|v\| \leq 1, \quad\|u-v\|>\varepsilon .
$$

Then it follows from the unit ball property in $X$ (see (8.4) ) that

$$
A_{f}(u) \leq 1, \quad A_{f}(v) \leq 1 .
$$

Since $\left\|\frac{u-v}{2}\right\|>\frac{\varepsilon}{2}$, we have

$$
A_{f}\left(\frac{u-v}{2}\right)>\tau \stackrel{10.2}{\geq} \tau \frac{1}{2}\left(A_{f}(u)+A_{f}(v)\right)
$$

for the corresponding $\tau=\tau(\varepsilon)>0$ by property (i); moreover, the proof of this property shows that $\tau(\varepsilon) \rightarrow 0$ as $\varepsilon \rightarrow 0$. It follows from the uniform convexity of the functional $A_{f}(\cdot)$ that there exists a $\sigma=\sigma(\tau)>0$ such that

$$
A_{f}\left(\frac{u+v}{2}\right) \leq(1-\sigma) \frac{1}{2}\left(A_{f}(u)+A_{f}(v)\right) \stackrel{\sqrt{10.2}}{\leq} 1-\sigma .
$$

Hence by property (ii) we have $\left\|\frac{u+v}{2}\right\| \leq 1-\delta$ for the corresponding $\delta=\delta(\varepsilon)>0$, as desired.

It remains to verify properties (i) and (ii). Let $2^{-j} \leq \varepsilon$, and set $\delta=K^{-j}$, where $K$ is the constant in the $\Delta_{2}$-condition, $A_{f}(2 v) \leq K A_{f}(v)$. Then $A_{f}\left(2^{j} v\right) \leq K^{j} A_{f}(v) \leq$ 
$K^{j} \delta=1$ provided that $A_{f}(v) \leq \delta$. It follows by the definition of the norm that $\|v\|_{L^{f}} \leq$ $2^{-j} \leq \varepsilon$, which is just what is needed for property (i).

Now take a $t \in(1,2)$. Owing to the representation

$$
t v=(2-t) v+(t-1) 2 v, \quad \text { where }(2-t)+(t-1)=1,2-t>0, t-1>0,
$$

it follows from convexity and the $\Delta_{2}$-condition that

$$
A_{f}(t v) \leq(2-t) A_{f}(v)+(t-1) A_{f}(2 v) \leq[(2-t)+K(t-1)] A_{f}(v),
$$

where the constant in square brackets is as close to unity as desired for $t$ sufficiently close to unity. Using the condition $A_{f}(v) \leq 1-\varepsilon, \varepsilon>0$, one can ensure that the right-hand side of (10.3) does not exceed unity, and then $A_{f}(t v) \leq 1$. By the definition of the norm, $\|v\|_{L^{f}} \leq t^{-1}=1-\delta$ for some $\delta=\delta(\varepsilon)>0$, which is just what is needed for property (ii).

The proof of Theorem 8.1 has been carried out following the scheme of proof of its counterpart [13, Theorem 2.4.14].

\section{REFERENCES}

[1] J. A. Clarkson, Uniformly convex spaces, Trans. Amer. Math. Soc. 40 (1936), no. 3, $396-414$. MR 1501880

[2] W. Rudin, Functional analysis, McGraw-Hill, New York, 1973. MR0365062 (51:1315)

[3] K. Yosida, Functional analysis, Springer, Berlin-Göttingen-Heidelberg, 1965.

[4] J. Diestel, Geometry of Banach spaces. Selected topics, Lect. Notes in Math., vol. 485, Springer, Berlin-Heidelberg-New York, 1975. MR0461094 (57:1079)

[5] J. Lindenstrauss and L. Tzafriri, Classical Banach spaces, Classics in Mathematics, Springer, Berlin, 1996. MR0415253 (54:3344)

[6] V. V. Zhikov, Passage to the limit in nonlinear variational problems, Mat. Sb. 183 (1992), no. 8, 47-84; English transl., Russ. Acad. Sci. Sb. Math. 76 (1993), no. 2, 427-459. MR1187249(93i:49016)

[7] V. V. Zhikov, On variational problems and nonlinear elliptic equations with nonstandard growth conditions, Probl. Mat. Analiza 54 (2011), 23-112; English transl., J. Math. Sci. (N. Y.) 173 (2011), no. 5, 463-570. MR2839881(2012i:35058)

[8] A. Braides, Г-convergence for beginners, Oxford Lecture Series in Math. and Its Appl., vol. 22, Oxford University Press, Oxford, 2002. MR1968440 (2004e:49001)

[9] V. V. Zhikov, The Lavrent'ev effect and averaging of nonlinear variational problems, Differentsial'nye Uravneniya 27 (1991), no. 1, 42-50; English transl., Differential Equations 27 (1991), no. 1, 32-39. MR1133509 (92j:49006)

[10] S. E. Pastukhova and A. S. Khripunova, Some versions of the compensated compactness principle, Mat. Sb. 202 (2011), no. 9, 135-160; English transl., Sb. Math. 202 (2011), no. 9-10, 1387-1412. MR.2884368

[11] V. V. Jikov, S. M. Kozlov, and O. A. Olejnik, Homogenization of differential operators and integral functionals, Nauka, Moscow, 1993; English transl., Springer, Berlin, 1994. MR1329546 (96h:35003b)

[12] S. E. Pastukhova and A. S. Khripunova, Gamma-closure of some classes of nonstandard convex integrands, Probl. Mat. Analiza 59 (2011), 73-94; English transl., J. Math. Sci. (N. Y.) 177 (2011), no. 1, 83-108. MR2838988

[13] L. Diening, P. Harjulehto, P. Hästö, and M. Rǔžička, Lebesgue and Sobolev spaces with variable exponents, Lect. Notes in Math., vol. 2017, Springer, Berlin, 2011. MR2790542

[14] E. S. Polovinkin and M. V. Balashov, Elements of convex and strongly convex analysis, 2nd ed., Fizmatlit, Moscow, 2007. (Russian)

[15] I. Ekeland and R. Temam, Convex analysis and variational problems, Studies in Math. and Its Appl., vol. 1, North-Holland, Amsterdam-Oxford; copublished with Elsevier, New York, 1976. MR 0463994 (57:3931b)

[16] V. V. Zhikov and S. E. Pastukhova, The $\Gamma$-convergence of oscillating integrands with nonstandard coercivity and growth conditions, Mat. Sb. 205 (2014), no. 4, 33-68; English transl., Sb. Math. 205 (2014), no. 4, 488-521.

[17] V. V. Zhikov and S. E. Pastukhova, The $\Gamma$-convergence of integrands with nonstandard coercivity and growth conditions, Probl. Mat. Analiza, 74 (2013), 85-108. (Russian)

[18] J.-L. Lions, Quelques méthodes de résolution des problèmes aux limites non linéaires, Dunod, Paris; copublished with Gauthier-Villars, Paris, 1969. MR0259693 (41:4326) 
[19] V. V. Zhikov and S. E. Pastukhova, Homogenization of monotone operators under conditions of coercitivity and growth of variable order, Mat. Zametki 90 (2011), no. 1, 53-69; English transl., Math. Notes 90 (2011), no. 1-2, 48-63. MR2908169

A. G. and N. G. Stoletov Vladimir State University, Vladimir, Russia

E-mail address: zhikov@vlsu.ru

Moscow State Technical University of Radio Engineering, Electronics, and Automation, Moscow, Russia

E-mail address: pas-se@yandex.ru

Translated by V. E. NAZAIKINSKII

Originally published in Russian 